\title{
Management of patients with multidrug- resistant/extensively drug-resistant tuberculosis in Europe: a TBNET consensus statement
}

\author{
Christoph Lange, Ibrahim Abubakar, Jan-Willem C. Alffenaar, \\ Graham Bothamley, Jose A. Caminero, Anna Cristina C. Carvalho, \\ Kwok-Chiu Chang, Luigi Codecasa, Ana Correia, Valeriu Crudu, Peter Davies, \\ Martin Dedicoat, Francis Drobniewski, Raquel Duarte, Cordula Ehlers, \\ Connie Erkens, Delia Goletti, Gunar Günther, Elmira Ibraim, Beate Kampmann, \\ Liga Kuksa, Wiel de Lange, Frank van Leth, Jan van Lunzen, Alberto Matteelli, \\ Dick Menzies, Ignacio Monedero, Elvira Richter, Sabine Rüsch-Gerdes, \\ Andreas Sandgren, Anna Scardigli, Alena Skrahina, Enrico Tortoli, \\ Grigory Volchenkov, Dirk Wagner, Marieke J. van der Werf, Bhanu Williams, \\ Wing-Wai Yew, Jean-Pierre Zellweger and Daniela Maria Cirillo for the TBNET
}

Affiliations: For the authors' affiliations see the Acknowledgements section.

Correspondence: Christoph Lange, Division of Clinical Infectious Diseases, German Center for Infection Research (DZIF) Clinical Tuberculosis Center, Medical Clinic, Research Center Borstel, Parkallee 35, 23845 Borstel, Germany. E-mail: clangedfz-borstel.de

ABSTRACT The emergence of multidrug-resistant (MDR) and extensively drug-resistant (XDR) tuberculosis (TB) substantially challenges TB control, especially in the European Region of the World Health Organization, where the highest prevalence of MDR/XDR cases is reported. The current management of patients with MDR/XDR-TB is extremely complex for medical, social and public health systems. The treatment with currently available anti-TB therapies to achieve relapse-free cure is long and undermined by a high frequency of adverse drug events, suboptimal treatment adherence, high costs and low treatment success rates. Availability of optimal management for patients with MDR/XDR-TB is limited even in the European Region. In the absence of a preventive vaccine, more effective diagnostic tools and novel therapeutic interventions the control of MDR/XDR-TB will be extremely difficult. Despite recent scientific advances in MDR/XDR-TB care, decisions for the management of patients with MDR/XDR-TB and their contacts often rely on expert opinions, rather than on clinical evidence.

This document summarises the current knowledge on the prevention, diagnosis and treatment of adults and children with MDR/XDR-TB and their contacts, and provides expert consensus recommendations on questions where scientific evidence is still lacking.

TBNET consensus statement on the management of patients with MDR/XDR-TB has been released in the Eur Respir J http://ow.ly/uizRD

For editorial comments see page 5 .

Received: Oct 302013 | Accepted after revision: March 032014 | First published online: March 232014

Support statement: This work was supported by the EU FP7 project TBPANNET (D.M. Cirillo and C. Lange), the German Center for Infection Research (DZIF) (C. Lange), the German Federal Ministry of Education and Research (D. Wagner), the UK Medical Research Council (I. Abubakar and B. Kampmann) and National Institute for Medical Research (I. Abubakar), and the National Institute of Health Research (UK) (B. Kampmann).

Copyright CERS 2014. ERJ Open articles are open access and distributed under the terms of the Creative Commons Attribution Non-Commercial Licence 4.0. 


\section{Introduction}

Tuberculosis (TB) is a leading cause of morbidity and ranks among the 10 most common causes of death worldwide [1]. The emergence of drug-resistant strains of Mycobacterium tuberculosis is substantially challenging the goal of elimination of TB in the 21 st century. The estimated global number of multidrugresistant (MDR) and extensively drug-resistant (XDR)-TB cases among newly diagnosed patients with pulmonary TB in the year 2012 was 450000 (range 300 000-600 000) [2]. Only 28\% of these estimated $\mathrm{MDR} / \mathrm{XDR}-\mathrm{TB}$ cases are notified, and the majority of these cases are from eastern European and central Asian countries [2]. Out of 83714 notified cases of pulmonary MDR/XDR-TB in the year 2012 worldwide, $36700(44 \%)$ cases were from the World Health Organization (WHO) European Region [2]. At least 92 countries have now reported patients with XDR-TB and the proportion of MDR-TB cases with XDR-TB is highest in the WHO European Region [2]. A recent study showed that $35.3 \%$ of patients with a first episode of TB and $76.5 \%$ of patients with a new TB episode who had been previously treated for TB from Minsk, Belarus, were diagnosed with MDR/XDR-TB [3]. This observation was also made countrywide for Belarus and represents the highest rate of MDR/XDR-TB for a country ever recorded [4].

Treatment success rates of MDR/XDR-TB vary between 36\% and 79\% [5, 6]. The 2013 joint WHO and European Centre for Disease Prevention and Control (ECDC) surveillance report recorded only 31.6\% successful treatment outcome for MDR/XDR-TB cases in the European Union (EU)/European Economic Area (EEA) region [7], comparable to the situation in the pre-antibiotic era [8]. It is estimated that, globally, $<20 \%$ of patients with pulmonary MDR/XDR-TB are currently receiving adequate treatment [2].

Infectious diseases caused by mycobacteria differ from almost all other bacterial infections by the requirement for a very long duration of treatment in order to achieve relapse-free cure with the currently available therapies. This is especially the case for TB when M. tuberculosis is MDR (i.e. resistant to the most potent anti-TB drugs rifampicin and isoniazid) [9]. The current recommendation from WHO for the duration of treatment of patients with MDR/XDR-TB is for a period of 20 months with a combination of four or more anti-TB drugs to which the organism is sensitive [10]. Suboptimal treatment adherence, adverse drug events and high treatment costs during the long course of therapy in MDR/XDR-TB severely compromise treatment success $[11,12]$.

Major advances have been achieved in recent years for the rapid identification of patients with MDR/XDRTB $[13,14]$, but these technologies are not yet universally available and developments in the area of diagnostics do not match the developments in therapeutics or prevention. However, for the first time in more than four decades, a new drug for the treatment of TB was licensed by the US Food and Drug Administration for the treatment against TB (specifically for the treatment of MDR/XDR-TB) at the end of 2012 [15], and following a positive recommendation by the European Medicines Agency (EMA) at the end of 2013, authorisation for the European market for two new antituberculosis drugs is expected soon.

In response to the alarming problem of MDR/XDR-TB, all 53 member states of the WHO European Region have fully endorsed the Consolidated Action Plan to Prevent and Combat MDR/XDR-TB in the WHO European Region 2011-2015, and its accompanying resolution at the 61st session of the WHO Regional Committee for Europe. This Plan has six strategic directions and seven areas of intervention, and aims to contain the spread of drug-resistant TB by achieving universal access to prevention, diagnosis and treatment of MDR/XDR-TB in all Member States of the Region [16].

Up to now, many issues in the management of patients with MDR/XDR-TB rely on expert opinions rather than on evidence. Examples are the evaluation of the duration of infectiveness of the patients, the choice of preventive chemotherapy for contacts of patients with MDR/XDR-TB [17], the optimal duration of therapy in individual patients and the best combination of anti-TB drugs given the drug resistance pattern of M. tuberculosis, to name but a few.

\section{Methodology}

This document has been developed predominantly by physicians treating patients with MDR/XDR-TB to reach a consensus about difficult management issues relating to the care of these patients. We did not intended to perform a systematic review of the literature with evidence grading or to substitute for recommendations by the WHO or ECDC. Following planning of the document structure by the coordinating authors (C. Lange and D.M. Cirillo) and agreement by the TBNET steering committee,

This article has supplementary material available from www.erj.ersjournals.com

A press release for this article is available from www.erj.ersjournals.com/site/misc/presspack.xhtml

Conflict of interest: Disclosures can be found alongside the online version of this article at www.erj.ersjournals.com 
co-authors were invited from among international experts in the field of MDR/XDR-TB (chapter leaders and authors are identified in the Acknowledgements section). Review of the available literature was accomplished by the members of the writing committee and the search for evidence included handsearching journals, reviewing previous guidelines, and searching electronic databases including MEDLINE and PubMed. Final decisions for formulating recommendations were based upon the result of literature review and practical experience by committee members.

The coordinating authors met on a regular basis while communication between co-authors and exchange of manuscript drafts was performed by e-mail correspondence. Each chapter was reviewed in a three-step process by the chapter co-authors, coordinating authors and all manuscript authors. Consensus statements (chapter 20) were developed in a stepwise approach.

Step 1

Preliminary proposals for 15 key recommendations were drafted by the coordinating authors. Chapter leaders were asked to provide alternative statements following consultation with the co-authors of their chapter.

\section{Step 2}

Alternative statements were collected by the coordinating authors.

Step 3

Chapter leaders were asked to select one preferred statement among the alternative statements following consultation with the co-authors of their chapter.

\section{Step 4}

For each recommendation, the statement that received most votes was selected for inclusion in the manuscript.

\section{Step 5}

All co-authors were asked to indicate their agreement, disagreement or whether they preferred to abstain from a decision on a recommendation. Results of the decisions are indicated at the end of each consensus statement.

\section{Definitions and reference documents}

\subsection{Case definitions}

Resistance to anti-TB drugs is identified through laboratory tests. Both phenotypic methods that involve culturing $M$. tuberculosis in the presence of anti-TB drugs and genotypic methods that identify specific mutations in the genome of the bacteria associated with resistance against individual drugs can be used. Table 1 provides the definitions of MDR-TB and XDR-TB, based on the results of bacteriological methods [18]. Recently, WHO has revised the case definitions of TB and drug-resistant TB so that also the results of WHO-endorsed new molecular and nonmolecular methods can be used to define a case. Case definitions were furthermore revised to avoid judgmental language, so the terms "defaulter" and "TB suspect" have been replaced by "lost to follow-up" and "presumptive TB", respectively [18].

From 2006 onwards, strains were reported with resistance to all first-line and second-line anti-TB drugs that were tested [19-21]. Experts recommended not to define these strains as totally drug-resistant or extremely drug-resistant TB because of technical difficulties with drug susceptibility testing (DST) of many anti-TB medicines, insufficient evidence to link such DST results to treatment outcomes of patients and the lack of standardised DST methods for several anti-TB drugs [22].

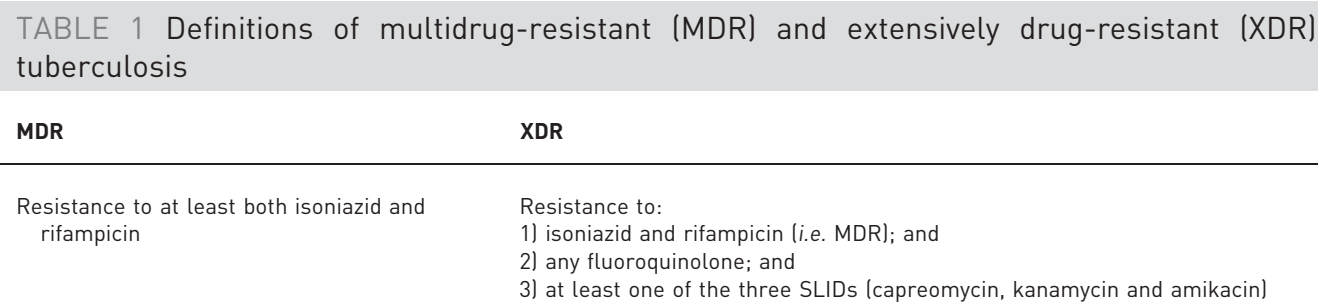

SLID: second-line injectable drug. Information from [18]. 


\section{TABLE 2 Treatment outcome categories and definitions for multidrug-resistant/extensively drug-resistant tuberculosis (TB)} patients

\begin{tabular}{|c|c|}
\hline Treatment outcome category & Definition \\
\hline Cured & $\begin{array}{l}\text { Treatment completed as recommended by the national policy without evidence of failure, and } \\
\text { three or more consecutive cultures taken } \geqslant 30 \text { days apart are negative after the intensive phase }\end{array}$ \\
\hline Treatment completed & $\begin{array}{l}\text { Treatment completed as recommended by the national policy without evidence of failure, but } \\
\text { no record that three or more consecutive cultures taken } \geqslant 30 \text { days apart are negative after the intensive phase } e^{\#}\end{array}$ \\
\hline Treatment failed & $\begin{array}{l}\text { Treatment terminated or need for permanent regimen change of at least two anti-TB drugs because of } \\
\text { lack of conversion by the end of the intensive phase } e^{\#} \text {, or } \\
\text { bacteriological reversion in the continuation phase after conversion }{ }^{\#} \text { to negative, or } \\
\text { evidence of additional acquired resistance to fluoroquinolones or SLIDs, or } \\
\text { adverse drug reactions }\end{array}$ \\
\hline Died & A patient who dies for any reason during the course of treatment \\
\hline Lost to follow-up & A patient whose treatment was interrupted for two consecutive months or more (this category was previously known as "defaulted") \\
\hline Not evaluated & $\begin{array}{l}\text { A patient for whom no treatment outcome is assigned (this includes cases "transferred out" to another treatment unit and whose } \\
\text { treatment outcome is unknown) }\end{array}$ \\
\hline
\end{tabular}

SLID: second-line injectable drug. ": for "treatment failed", lack of conversion by the end of the intensive phase implies that the patient does not convert within the maximum duration of intensive phase applied by the programme. If no maximum duration is defined, an 8-month cut-off is proposed. For regimens without a clear distinction between intensive and continuation phases, a cut-off 8 months after the start of treatment is suggested to determine when the criteria for "cured", "treatment completed" and "treatment failed" start to apply. ": the terms "conversion" and "reversion" of culture as used here are defined as follows. Conversion (to negative): culture is considered to have converted to negative when two consecutive cultures, taken $\geqslant 30$ days apart, are found to be negative. In such cases, the specimen collection date of the first negative culture is used as the date of conversion. Reversion (to positive): culture is considered to have reverted to positive when, after an initial conversion, two consecutive cultures, taken $\geqslant 30$ days apart, are found to be positive. For the purpose of defining "treatment failed", reversion is only considered when it occurs in the continuation phase. Reproduced and modified from [18] with permission from the publisher.

\subsection{Treatment outcome definitions}

Recently, WHO revised the MDR-TB treatment outcome definitions (table 2) [18]. The aim was to simplify the outcome definitions for MDR-TB cases and to make the treatment outcomes useful for patient care decision making.

\subsection{Reference documents}

WHO has published guidelines for the programmatic management of drug-resistant TB. The 2011 updated guidelines address the most topical questions concerning the programmatic management of drug-resistant TB [10]. The emergency update of 2008 provided recommendations on how to manage drug-resistant TB [23]. This update is accompanied by a field guide that is intended to assist health workers [24]. Recently, the International Union against Tuberculosis and Lung Disease (the Union) also issued guidelines on the management of MDR-TB [25].

In 2012, the European Union Standards for Tuberculosis Care (ESTC) were published [26]. These standards discuss diagnosis and treatment of $\mathrm{TB}$ and related public health issues. In addition to the International Standards of Tuberculosis Care (ISTC) [27], the ESTC recommend rapid testing for the identification of rifampicin and isoniazid resistance in settings in which MDR-TB is suspected in a patient and the application of second-line DST.

Other than these general guidelines, more specific reference documents have been published. Two documents specifically discuss TB contact investigation [28, 29]. Recommendations on treatment of latent infection with $M$. tuberculosis including treatment of contacts of MDR-TB patients have been published by the Centers for Disease Control and Prevention (CDC) [30]. The ECDC published a guidance document specifically on management of contacts of MDR-TB and XDR-TB patients [17].

\subsection{Conclusions}

1) TB case definitions and treatment outcome definitions were revised by WHO in March 2013.

2) Various guidelines relevant to management of patients with MDR/XDR-TB have been published by WHO, the Union, ECDC, CDC, the European Respiratory Society (ERS), ISTC and ESTC.

\section{Epidemiology of MDR/XDR-TB in Europe}

15 of the 27 high MDR-TB burden countries worldwide are in the European Region [31]. In 2011, 49 of the 53 countries of the WHO European Region reported on first-line anti-TB DST; 89877 new TB cases and 31929 previously treated TB cases were subjected to DST to first-line anti-TB drugs. In 2011, the prevalence of MDR-TB was $13.2 \%$ among new cases and $46.4 \%$ among previously treated cases, respectively [7]. In 2011, nine countries had a MDR-TB rate of 10-30\% among all TB cases and in five countries this rate was $>30 \%$ (fig. 1). These countries are Belarus (52.6\%), Kazakhstan (39.6\%), the Republic of Moldova (42.0\%), Tajikistan (66.0\%) and Uzbekistan (62.5\%) [7]. The data from two of these countries (Kazakhstan 


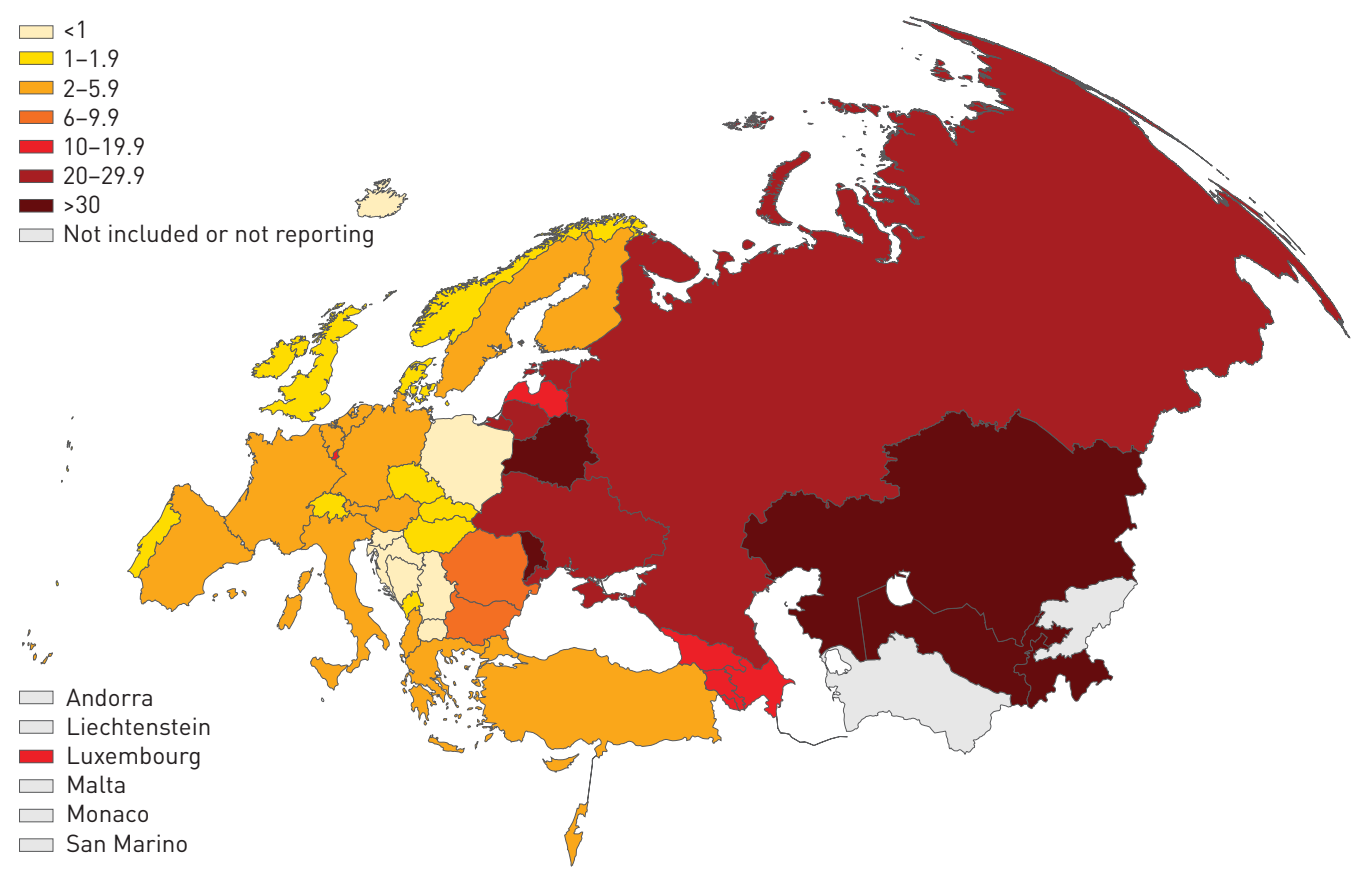

FIGURE 1 Percentage of notified tuberculosis (TB) cases with multidrug resistance among all TB cases with drug susceptibility results in the World Health Organization European Region, 2011. Note that the data from Azerbaijan, Italy, Kyrgyzstan, Serbia, Spain, Tajikistan, Turkey, Turkmenistan and Uzbekistan cover only part of the country, and may therefore not be representative and should be interpreted with care. In addition, note that data from the United Nations Administrated Province of Kosovo (in accordance with Security Council Resolution 1244 (1999)) is not included in the figures reported for Serbia. Reproduced from [7] with permission from the publisher.

and Tajikistan) are based on partial national data only and should be interpreted with caution. The prevalence of MDR-TB among new cases was $\leqslant 4 \%$ in all EU/EEA countries, except for the Baltic states, where this prevalence ranged from $11.1 \%$ in Lithuania to $22.9 \%$ in Estonia. Owing to limited DST to second-line drugs (SLDs), data on the extent of XDR-TB are limited. WHO estimated that $\sim 8100$ XDR-TB cases emerged world-wide in 2010 [31]. The vast majority of reported XDR-TB cases reported occurred in the Eastern part of the Region. Of the 3448 MDR-TB cases tested for SLD resistance in 2011, 381 (11.0\%) were XDR [7]. The MDR-TB epidemic in the Eastern part of the region coincides with an increasing HIV prevalence; HIV co-infection among TB cases increased from $3.6 \%$ in 2008 to $6.2 \%$ in 2011 [7].

In the last decade, the proportion of MDR-TB cases increased steadily in the European Region; it tripled among previously treated cases and increased five-fold among new cases. These data suggest active transmission of MDR-TB. According to the latest ECDC report, out of the 78000 (range 31 000-85000) estimated MDR-TB cases in the region, only 30 000 MDR-TB cases were notified in 2011, mainly because of limited laboratory capacity [7]. These estimated 78000 MDR-TB cases represent $25 \%$ of the 310000 (range 220000-400 000) MDR-TB cases estimated to occur globally in 2011, whereas the number of new and relapse $\mathrm{TB}$ cases in the region $(380000)$ only represents just $4.4 \%$ of the global burden of $\mathrm{TB}$, underscoring the specific problems with MDR-TB in the WHO European Region [7, 32]. The estimated number of patients with MDR/XDR-TB has increased again in 2012 to 450000 (range 300000-600 000) globally [2].

\section{1 Conclusions}

1) Drug-resistant TB is a major challenge to controlling the TB epidemic in the WHO European Region due to the alarmingly high incidence of MDR-TB; 15 of the 27 high MDR-TB burden countries worldwide are in this region.

2) The problem of MDR-TB is most pronounced in the WHO European Region; whereas the number of TB cases in the region represents $<5 \%$ of the global TB burden, the estimated MDR-TB burden represents $25 \%$ of the global burden.

3) In 2012, the prevalence of MDR-TB in the WHO European Region was 16\% (range 10-22\%) among new cases and 45\% (range 39-52\%) among previously treated cases, respectively. 
TABLE 3 Risk factors for multidrug-resistant (MDR)/extensively drug-resistant (XDR) tuberculosis (TB)

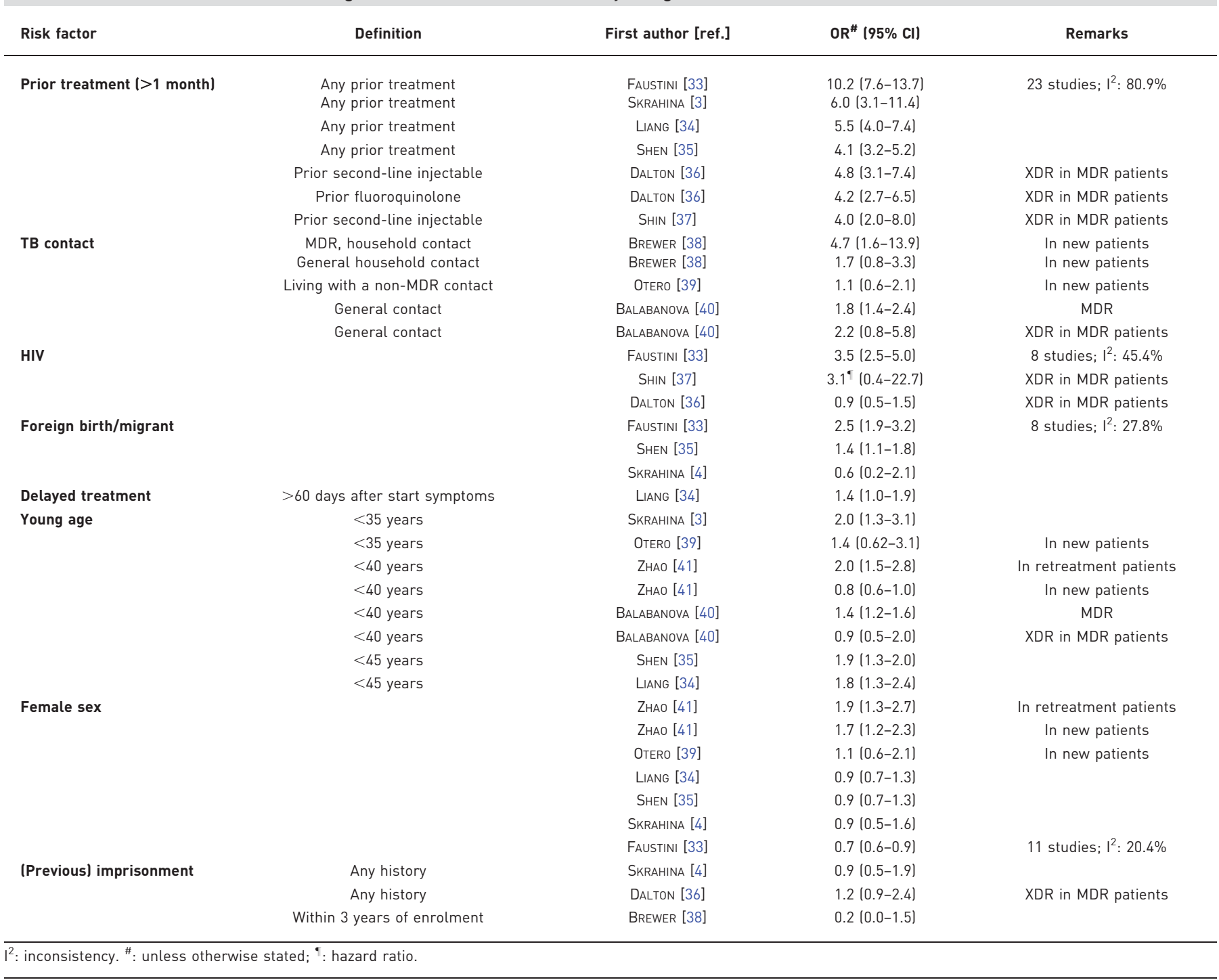

4) The absolute number of MDR-TB among new TB patients is higher than among previously treated patients. In these patients, the presence of drug-resistant strains is frequently unsuspected. The increasing proportion of MDR-TB among new patients is a matter of great concern in many countries as it suggests active transmission of strains MDR/XDR strains of M. tuberculosis.

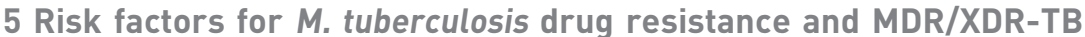

While a formal systematic review would have been beyond the scope of this document, a selection of risk factors for MDR-TB, based on in-depth knowledge of the literature for this topic, is reported in table 3. Among them, prior TB treatment for $>1$ month is the most important. This could particularly be the case if the treatment was inappropriate for the drug susceptibility of the strain [42], although the evidence is scarce. Other prominent risk factors include close contact to a patient with MDR-TB, migration, HIV infection and young age. TB contact and previous imprisonment showed an increased risk for XDR-TB in patients with MDR-TB, although this trend is not statistically significant due to the low number of patients, even in the larger studies [36, 40].

Early discontinuation of adequate therapy is a risk factor for relapse but does not seem to induce drug resistance [43]. On the contrary, failure of treatment or relapse after therapy may be the consequence of the use of an inappropriate treatment against MDR/XDR-TB or with the causative M. tuberculosis strain [44]. It can be postulated that inadequate quality of anti-TB drugs and low drug exposure contribute to the development of drug resistance in M. tuberculosis [45, 46]. 
It is alarming that MDR/XDR-TB is now observed in a substantial proportion of patients without risk factors for MDR/XDR-TB, other than living in an area of high MDR/XDR-TB prevalence in Europe [4, 39], or in new patients who have never been treated for TB [47]. This indicates active transmission of drug-resistant strains in these countries, with an important role for imprisonment [48] and hospitalisation [41], possibly because of insufficient infection control measures in these facilities.

Important for clinical management is the possibility that strains of M. tuberculosis may have acquired additional drug resistance beyond isoniazid and rifampicin. A prospective cohort study in eight countries demonstrated that bacterial strains of $43.7 \%$ of patients with MDR-TB already showed resistance against at least one SLD, $20.0 \%$ against one injectable drug and $12.9 \%$ against fluoroquinolones [36]. Previous treatment with SLDs was the strongest risk factor for the development of resistance against these drugs, thus increasing the risk of XDR-TB. A study in Russia demonstrated that $6 \%$ of patients with MDR-TB developed XDR-TB during TB treatment [49]. The risk factors were extensive lesions, the prior use of injectable SLDs and long duration of inappropriate treatment with insufficient intake of the medication.

In most Eastern European countries, MDR/XDR-TB must be highly suspected in all patients who were treated for TB in the past. However, as transmission of MDR/XDR M. tuberculosis strains in the community is high, all patients with TB in these countries must be evaluated for MDR/XDR-TB by rapid diagnostics. In Western European countries, MDR/XDR-TB must be considered especially in migrants with TB coming from high MDR/XDR-TB prevalence countries. According to unpublished data from Switzerland, $90 \%$ of MDR-TB cases were observed among foreign-born patients, who represent some $20 \%$ of the local population. Among MDR-TB patients, the absolute number of new cases was higher than previously treated patients. Although never investigated in detail, the risk of MDR/XDR-TB is probably very high in those patients who migrate to other countries for the purpose of seeking TB treatment [50].

\subsection{Conclusions}

1) The risk of MDR-TB is higher among patients who already received prior treatment with anti-TB drugs and in patients living in countries with a high rate of primary MDR-TB.

2) The risk of further resistance to SLDs is higher in patients previously exposed to those drugs, especially if under inappropriate regimens.

3) In countries with a high burden of $M D R / X D R-T B$, the suspicion of primary drug resistance to M. tuberculosis is high in all patients with TB, even when no risk factors or prior treatment are present.

4) Although, as yet, evidence is lacking, it seems that in countries with a low prevalence of MDR/XDR-TB, healthcare-seeking migrants with TB and migrants from countries with a high prevalence of MDR-TB probably have a high risk of MDR/XDR-TB.

\section{Contact investigations and preventive chemotherapy in MDR/XDR-TB contacts}

Molecular epidemiology studies suggest that, in some settings, the majority of MDR/XDR-TB cases are related to ongoing transmission of MDR strains of M. tuberculosis [51]. This emphasises the need for active case finding, rapid diagnosis, comprehensive contact tracing and infection control in order to reduce disease transmission within the community $[52,53]$. The consequences of acquiring an infection with MDR/XDR-TB are more serious than infection with a drug-susceptible strain. This warrants high priority for the screening of close contacts of MDR/XDR-TB patients [54, 55].

The need to screen contacts should be assessed on the basis of 1) the infectiousness of the index case, 2) intensity of exposure and 3) susceptibility of contacts to TB [28]. Patients with acid-fast bacilli (AFB) sputum smear-positive TB are most infectious. Intensity of exposure is determined by proximity to the index patient, duration of exposure, and the physical conditions of the location where transmission may have occurred in terms of shared breathing space, ventilation and ambient light. Consequently, household contacts and contacts sharing breathing space for prolonged periods are most at risk. Young children and immunocompromised persons have a higher risk of developing TB [52]. Contact investigation should include all close contacts and may be expanded to lower risk contacts $[28,56]$ as recommended in national guidelines.

Following an incubation period of about 6-8 weeks, the risk of progression to TB is highest shortly after infection, followed by an exponential decline in subsequent years $[57,58]$. Therefore, contacts should be evaluated for infection with M. tuberculosis with the tuberculin skin test (TST) and/or an interferon- $\gamma$ release assay (IGRA) $\geqslant 8$ weeks after the last infectious exposure. Immunocompromised contacts, children and contacts with symptoms suggestive of TB should be evaluated as soon as possible after diagnosis of the index case. If a contact is symptomatic, or considered to be infected, TB disease should be excluded. In children $<5$ years of age and immunocompromised persons, TB disease should be excluded, irrespective of 
the TST or IGRA response. All contacts or caretakers should receive information regarding the likelihood of latent infection with M. tuberculosis (LTBI) developing into TB disease and symptoms of TB disease.

The evidence for the effectiveness of chemoprophylaxis or preventive therapy of MDR/XDR-TB contacts when infected is scarce [59, 60], although there are indications of a positive effectiveness in children [61] and in adults $[62,63]$. Given the lack of evidence from controlled clinical trials, both preventive treatment and careful clinical follow-up are valid options for the management of contacts, provided they are informed of the benefits and harm $[17,59,64]$. Special attention with regard to individual risk assessment should be paid to children $<5$ years of age and immunocompromised persons, particularly those with evidence of LTBI. If chemoprophylaxis or preventive treatment is considered, the drug regimen is dictated by the drugresistance pattern of the source case, whether a drug with bactericidal activity is available, the likelihood of adherence and the risk of adverse events. There are no data defining the minimum duration of such a preventive therapy or whether it is safe to give the drug as monotherapy. Therefore, until further data are available, monotherapy may be inadvisable. It is essential to monitor adverse events closely and to provide support to ensure adherence during preventive treatment. Chemoprophylaxis may be discontinued if, based on further examination, infection is found to be unlikely.

When preventive therapy is not an option, or risks outweigh the benefits, contacts and their general practitioners should be educated about the risks of progressing to TB disease. Temporary periodical followup can be considered, but easy access to a specialised TB clinic in case symptoms appear is of higher importance $[65,66]$.

\subsection{Conclusions}

1) Screening of close contacts of MDR/XDR-TB patients to prevent morbidity and further transmission warrants high priority.

2) Contacts or caretakers should be informed regarding likelihood of LTBI developing into MDR/XDR-TB disease and symptoms of active TB.

3) Evidence for the effectiveness of preventive therapy of infected MDR/XDR-TB contacts is low and is currently based on expert opinions. Currently, there is insufficient evidence to recommend preventive chemotherapy of MDR/XDR-TB contacts in general, and the optimal duration of preventive chemotherapy is unknown.

4) Preventive treatment should only be considered by a physician with expertise in this area after adequate exclusion of active disease and when a drug with bactericidal activity is available within the drug sensitivity pattern of the M. tuberculosis culture from the source case; the risk of MDR/XDR-TB in the contact outweighs the risk of adverse events; and support to monitor adverse events and to ensure adherence can be provided.

5) With presently available anti-TB medications, preventive chemotherapy against MDR/XDR-TB should be a combination of at least two drugs to which the M. tuberculosis strain is susceptible according to the DST. At least one of these drugs should be bactericidal.

\section{Infection control measures for MDR/XDR-TB}

Infection control measures, especially in congregate settings, are necessary to prevent transmission of M. tuberculosis. CDC and WHO have developed policies and guidelines on TB infection control [67, 68]. A toolbox with documents that assist in implementing the WHO policy is available from the TB CARE website [69]. When it comes to preventing transmission, there is no essential difference between MDR/ XDR-TB strains and other strains of M. tuberculosis [70, 71].

The persons to be protected are household contacts (e.g. family members), and, in a hospital setting, other patients, healthcare workers, cleaners, administrative staff, etc., and visitors. The best way to prevent transmission of M. tuberculosis is prompt diagnosis and start of effective treatment [72-74]. Contagiousness of the index case can be measured by investigating the sputum for AFB, but $\sim 15 \%$ of transmission in the community comes from sputum AFB smear-negative index cases [75]. There is a wide variability in contagiousness of AFB sputum smear-positive index cases [76, 77]. It must be considered that, in general, the duration of contagiousness of patients with MDR/XDR-TB is longer than that of patients with nonMDR/XDR-TB [78].

Individuals suspected of having TB should be separated from other patients and evaluated for TB without waiting in general areas. Hospitalisation should include airborne isolation precautions and be limited primarily to contagious AFB sputum smear-positive TB patients. Infectiousness is substantially reduced 
once a patient is on an adequate regimen and it is probably not necessary to keep a patient in hospital until their cultures become negative $[72,73]$.

In some settings, patients with MDR/XDR-TB are discharged from the hospital after 2 weeks of an adequate treatment [74, 79-81], although many centres require a negative result from three sputum cultures collected over a 14-day period in patients with MDR/XDR-TB before they are considered for discharge. At this time, the optimal time for safe discharge of patients with MDR/XDR-TB is not known.

In daily practice, strict isolation of a patient is often not possible, such as in a patient with ongoing nicotine addiction. Smoking is generally forbidden in patient rooms and patients with contagious TB, especially MDR/XDR-TB, should not use shared smoking shelters. If patients are unwilling or unable to stop smoking, they should smoke outside. There must be awareness that in-patients will socialise in the evenings, at nights and on weekends, when infection control measures by hospital staff cannot be strictly enforced.

Any M. tuberculosis transmission risk reduction programme should include the issues of administrative and environmental measures and personal respiratory protection [68]. In hospitals specialising in the care of patients with MDR/XDR-TB, M. tuberculosis transmission risk assessment should be conducted regularly within a TB infection control plan. In high-risk areas of $M$. tuberculosis transmission, procedures and staff should be identified for personal respiratory protection (e.g. education and training on donning respirators, including their seal checks, use, care and disposal); respirator fit tests (qualitative or quantitative) should be organised on an annual basis [81-83].

Hospital staff sharing air with contagious patients with MDR/XDR-TB should be wearing FFP2-certified respirators [84]. Use of surgical masks by contagious MDR/XDR-TB patients indoors reduces transmission risk by half [83]. Because of the very high air change rate outdoors, any recommendation to use masks for TB patients in the open air is not justified.

Natural ventilation should be maximised by appropriate architectural design and rational administrative support [85]. Sustainable environmental controls should be implemented for high-risk areas for TB transmission where administrative controls cannot further substantially reduce the risk. Mechanical ventilation should provide directional airflow, and negative pressure is required in isolation rooms and other high-risk areas. 12 air changes per hour $(\mathrm{ACH})$ are recommended for new facilities, but $6 \mathrm{ACH}$ may be acceptable for older facilities [86]. Contaminated air exhaust outlets should by located $\geqslant 8 \mathrm{~m}$ from windows, air intakes and occupied areas, or effectively decontaminated by in-duct ultraviolet germicidal irrigation (UVGI) or high-efficiency particulate air filtration. Upper room UVGI fixtures, professionally designed, installed and maintained, are an effective and sustainable option for limited-resource settings [87-89]. Air recirculation and room air cleaners are not recommended for high $M$. tuberculosis transmission risk areas. To ensure sustainability of engineering controls, their application should be rationally limited to high-risk areas; systems should be professionally designed, installed, commissioned and maintained.

As bronchoscopy and endotracheal intubation of patients with pulmonary TB pose a very high risk of transmission for all occupants in the room, a clear protocol of TB hygiene precautions should be available for these interventions in patients with pulmonary $\mathrm{TB}$ or those with an unclear diagnosis where pulmonary $\mathrm{TB}$ is part of the differential diagnosis. Indications for such procedures should be strictly limited; they should be performed in isolated, ideally negative pressure mechanically ventilated rooms with $12 \mathrm{ACH}$. All occupants should wear FFP2 or FFP3 respirators. If effective mechanical ventilation is not available, the whole room should receive UVGI after completion of the procedure with sufficient exposure. To help reduce the risk of contaminating a ventilator or discharging $M$. tuberculosis into the ambient air when mechanically ventilating (i.e. with a ventilator or manual resuscitator) a patient with suspected or confirmed TB, a bacterial filter should be placed in the patient's endotracheal tube (or at the expiratory side of the breathing circuit of a ventilator). In selecting a bacterial filter, preference should be given to models specified by the manufacturer to filter particles $\geqslant 0.2 \mu \mathrm{m}$ in size in both the unloaded and loaded states with a filter efficiency of $>95 \%$ (i.e. filter penetration of $<5 \%$ ) at the maximum design flow rates of the ventilator for the service life of the filter, as specified by the manufacturer [68].

Transport of contagious patients inside the hospital should be limited (avoid use of lifts, sitting in waiting areas, etc.). Transport of contagious patients outside the hospital should preferably be carried out in vehicles with a separate compartment for the patient and for accompanying persons. If weather permits, open windows allow very high air change rates inside the vehicle. At least a surgical mask or a respirator without an exhalation valve should be worn by the patient, and a FFP2 respirator worn by the driver and accompanying person during the journey.

Some of these measures recommended by the international airborne infection control expert community have no solid evidence base and there is still a lot of research to be done in this field. 


\subsection{Conclusions}

1) The best way to prevent transmission of MDR/XDR-TB is a rapid diagnosis (high level of suspicion and rapid DST) and the prompt start of an effective treatment.

2) Airborne infection control precautions should be taken in accordance with international guidelines with regard to the local situation, with reference to the resource setting, climate and building facilities.

\section{Principles and interpretation of M. tuberculosis DST}

Quality-assured bacteriological examination is an essential element for diagnosis and management of patients infected with susceptible or resistant TB bacteria. Phenotypic DST remains the mainstay for the detection of drug resistance in $M$. tuberculosis and is based on detection of bacterial growth in the presence of antibiotics. Traditional solid-media culture techniques are reliable and cheap but slow, with an overall turnaround time of 6-12 weeks when a patient specimen is taken. Automated liquid-media systems are faster (average turnaround time of 14-21 days) [90, 91], and are recommended for use even in low- and middle-income countries. Whatever the test used, results from the Supranational Reference Laboratory Network have clearly shown that, while DST for rifampicin, isoniazid, fluoroquinolones and second-line injectables is reliable in solid- and liquid-media systems, there is more controversy around the standardisation of DST for other drugs (ethambutol, pyrazinamide and other SLDs) [91-99]. The WHO policy of testing for SLD susceptibilities suggests testing MDR-TB isolates for sensitivity to amikacin, kanamycin, capreomycin and newer fluoroquinolones (moxifloxacin at two concentrations or ofloxacin/ levofloxacin plus high concentration of moxifloxacin). For fluoroquinolones, testing of the drug in use in the country is recommended.

Recently, with the insights from mycobacterial genomics, molecular techniques to detect antibiotic resistance have been established. They offer advantages like turnaround times of hours and the possibility of omitting microbiological culture. A prerequisite is the knowledge of specific genetic mutations that are undoubtedly associated with resistance. The development of drug resistance in M. tuberculosis isolates results from random genetic mutations in genes involved with the drug mechanism of action. Several molecular assays have been established, allowing the prediction of drug resistance in clinical M. tuberculosis isolates within one working day or less.

Rifampicin is the most important drug for the treatment of TB and is considered a surrogate marker of MDR for its frequent association (80-95\% of cases) with resistance to isoniazid [100, 101].

An 81 base pair region of the $r p o B$ gene (encoding the $\beta$-subunit of RNA polymerase) harbours mutations responsible for rifampicin resistance in $\sim 97 \%$ of resistant strains [102]. This makes rifampicin resistance the ideal target for molecular testing.

Isoniazid requires activation in vivo by catalase-peroxidase (KatG); it is therefore not surprising that mutations in the $k a t G$ gene cause the majority of isoniazid resistance (50-90\% of isoniazid-resistant strains) [103]. Mutations in other regions may also be responsible for resistance, primarily by mutations in the inhA promoter and coding region, which encodes the target of activated isoniazid. On average, 80-90\% of isoniazid-resistant strains present mutations in either $\mathrm{kat} G$ or inhA [104]. Mutations in inhA confer crossresistance to ethionamide while strains mutated in $k a t G$ may still retain ethionamide sensitivity [105]. The different mutations confer different levels of resistance to isoniazid. In some cases, a low level of resistance can be overcome by the use of higher doses of isoniazid [106, 107] and it is therefore worth clarifying the resistance level with the microbiological laboratory.

For other first-line drugs (FLDs), suboptimal correlation or disagreement exists in the interpretation of the results: mutations in codon 306 of $e m b B$ are present in 47-62\% of ethambutol-resistant strains [108, 109], mutations in $r p s L$ (encoding ribosomal protein S12) are present in 54\% of the streptomycin-resistant strains and mutations in pncA (encoding pyrazinamidase) characterise $72-97 \%$ of pyrazinamide-resistant strains [110]. In some cases, the disagreement between phenotypic and genotypic results is due to the suboptimal performance of in vitro DST. The main mutations responsible for resistance identified for SLDs are in the gyrA gene (encoding a DNA gyrase) for fluoroquinolones (60-70\%) [111, 112], and in the rrs (encoding the $16 \mathrm{~S}$ ribosomal RNA) and eis (conferring enhanced intracellular survival) genes for injectable drugs [113, 114].

Gene sequencing is the gold standard for mutation detection. The current commercial PCR-based methods focus mainly on the detection of rifampicin resistance. They target the hot-spot region for mutations of the $r p o B$ gene and include two line probe assays (LPAs) (INNO-LiPA Rif.TB (Fujirebio, Ghent, Belgium) and GenoType MTBDRplus (Hain Lifescience GmbH, Nehren, Germany)) and the Xpert MTB/RIF system (Cepheid, Sunnyvale, CA, USA). Very recent data have shown that testing for rifampicin in liquid media may produce false-negative results due to the low growth rate of strains harbouring specific mutations in 
$r p o B$ [115]. These uncommon mutations are well identified by the Xpert MTB/RIF system but their presence needs to be confirmed by $r p o B$ gene sequencing, not by liquid culture.

Among the genetic tests, the LPAs are best suited for the rapid diagnosis of MDR-TB in reference or wellequipped laboratories from sputum smear-positive samples or strains isolated in culture. Molecular assays are highly accurate for identifying rifampicin resistance, as the mechanism of resistance is known. The detection rate of isoniazid resistance is lower due to the heterogeneity of the molecular biology background of this resistance.

Although the LPAs are endorsed by WHO for the rapid detection of MDR in smear-positive samples only, the newer generation tests show a high sensitivity in smear negative respiratory samples [110].

The commercially available LPA GenoTypeMTBDRsl aims to detect resistance to ethambutol, the secondline injectable drugs (SLIDs) and the fluoroquinolones. Because of the low negative predictive value (NPV) (probes included only detect a fraction of mutations known to cause resistance [109]), the test can only "rule in" XDR-TB and has not been endorsed by WHO [91]. Newer generations of tests targeting a larger number of mutated genes may overcome this problem in the future.

The only completely automated test able to detect simultaneously the presence of $M$. tuberculosis complex DNA and rifampicin resistance in clinical samples is the Xpert MTB/RIF assay [13, 117]. This assay simplifies molecular testing by fully integrating and automating the whole process required for sample preparation, real-time PCR and result interpretation. The Xpert MTB/RIF assay is currently the only technology suitable for near-patient use and it is recommended by WHO as the first test when MDR-TB is suspected and where TB is suspected in HIV-infected individuals [118]. Sensitivity and specificity for the detection of TB and MDR-TB in both pulmonary and extrapulmonary samples are high [119]. If the test is used in a low-prevalence MDR-TB population to test subjects not considered at risk for MDR-TB, rifampicin-resistant cases should be reconfirmed by a different rapid test or liquid culture. A second Xpert MTB/RIF test on a different sample, although not optimal, could be considered as a fast re-testing strategy.

\subsection{Conclusions}

1) Phenotypic DST remains currently the mainstay for the detection of drug resistance in M. tuberculosis.

2) Genotypic tests significantly decrease the time required for the determination of anti-TB drug susceptibility of $M$. tuberculosis. The tests for detection of rifampicin resistance are the most reliable (sensitive and specific, if used on a population at high risk for MDR-TB), while the detection of isoniazid resistance by LPAs is highly specific but the NPV is low due to multifactorial mechanisms causing resistance.

3) LPAs are relatively specific but unsatisfactory sensitivity affects the molecular tests for resistance to quinolones and, even more so, to SLIDs.

4) At present, there is poor agreement between phenotypic and genotypic tests, and response to therapy for other drugs (e.g. WHO group 4 and 5).

5) Patients with presumptive TB with significant risk factors for MDR/XDR-TB (such as those with a history of past treatment, contacts of MDR/XDR-TB patients and immigrants from high MDR-TB settings) should have direct molecular assays performed for detection of rifampicin resistance. If mutations are detected, the testing for FLDs and SLDs should be set up as soon as the liquid culture is positive for M. tuberculosis and rifampicin resistance, in order to shorten considerably the time to obtain results. Rapid molecular tests should be extended to HIV-infected patients with presumptive TB because of the limited diagnostic capacity of other diagnostic test for such cases and the high fatality rates associated with MDR/ XDR-TB in this patient group.

6) In MDR-TB low-prevalence settings, rifampicin resistance should be reconfirmed by one of the following: $r p o B$ sequencing, an independent molecular test, liquid culture or treatment of patients with MDR/XDR-TB.

\section{Treatment of patients with MDR/XDR-TB}

Treating drug-resistant TB, especially rifampicin-resistant $\mathrm{TB}$, has become a major challenge for TB control worldwide. With high bactericidal and sterilising activities, and low toxicity, rifampicin is by far the most active TB drug. As rifampicin monoresistance is relatively rare, except in HIV-infected patients, it serves as a surrogate marker of MDR-TB [120]. Among SLDs, newer-generation fluoroquinolones have demonstrated prominent bactericidal [121] and sterilising activities [122]. Bacillary resistance to fluoroquinolones clearly influences the prognosis in XDR-TB $[123,124]$. 
Regardless of the drug-resistance pattern, all TB forms should be treated with a combination of drugs for a sufficient duration to avoid relapse. The number of drugs in a multidrug regimen and the total treatment duration depend on the bactericidal and sterilising activities of the TB drugs included [123, 125]. Availability of drugs that are likely to be effective decreases as drug resistance becomes more extensive.

\subsection{Number of drugs for treating MDR/XDR-TB}

If $M$. tuberculosis is fully susceptible to all the TB drugs used in a regimen, two active core drugs, such as isoniazid and rifampicin, are probably adequate for cure [123]. In the treatment of MDR-TB, WHO advises giving at least four drugs that are known or likely to be effective against the drug-resistant $M$. tuberculosis strain harboured by the patient, plus pyrazinamide (fig. 2) [126]. If possible, WHO group 2 (amikacin, capreomycin or kanamycin) and WHO group 3 (fluoroquinolones) should be core drugs with the rest (ethionamide/prothionamide and cycloserine) being accompanying drugs that may help protect the core drugs in the initial phase when bacillary load is high [124, 125].

\subsection{What are the drugs for the treatment of MDR/XDR-TB?}

Drugs not previously used by the patient or those with in vitro activity based on DST results are more likely to be effective. For this reason, a detailed drug history is important for formulating the MDR-TB regimen [127]. However, in many countries, $20-30 \%$ of the patients with MDR-TB never received TB drugs before and the choice has to be made according to the drug-resistance patterns common in the local settings. It is very important to highlight that the clinical reliability of DST (concordance between in vitro and in vivo activity) is very good for isoniazid and rifampicin, good for fluoroquinolones and SLIDs, and low or uncertain for the other drugs [127-129]. Drugs that are likely to be effective are then included in the MDR$\mathrm{TB}$ treatment regimen according to the hierarchy described in figure 2 and table 4 . It is not suggested to choose more than one drug from each of group 2 and group $3[120,151]$.

In cases of fluoroquinolone-resistant MDR-TB and XDR-TB, group 5 drugs, notably linezolid [152], and possibly high-dose isoniazid and clofazimine, are often required to optimise treatment outcomes. Linezolid is an oxazolidinone that has been used off-label in the treatment of fluoroquinolone-resistant MDR/XDRTB (table 4) [10, 106, 126, 151]. Despite high costs and substantial toxicity (especially neurotoxicity after

DST result of $M$. tuberculosis from the index patient known

\begin{tabular}{|c|c|}
\hline $\begin{array}{c}\text { Active TB, } \\
\text { close contact of a MDR/XDR-TB index patient }\end{array}$ & $\begin{array}{l}\text { Active TB, } \\
\text { rifampicin ( } \pm \text { isoniazid) drug resistance suggested } \\
\text { by rapid molecular testing or MDR-TB identified by } \\
\text { M. tuberculosis culture; DST results of culture pending }\end{array}$ \\
\hline$\downarrow$ & $\downarrow$ \\
\hline \multirow[t]{2}{*}{$\begin{array}{l}\text { Treat according to the DST result of } \\
\text { M. tuberculosis from the index patient }\end{array}$} & $\begin{array}{l}\text { Use a standardised regimen until culture-based DST } \\
\text { results become available }\end{array}$ \\
\hline & $\begin{array}{l}\text { 1. levofloxacin or moxifloxacin } \\
\text { 2. amikacin, capreomycin or kanamycin } \\
\text { 3. prothionamide/ethionamide } \\
\text { 4. cycloserine/terizidone } \\
\text { 5. ethambutol and pyrazinamide }\end{array}$ \\
\hline$\downarrow$ & $\downarrow$ \\
\hline \multicolumn{2}{|c|}{$\begin{array}{c}\text { Adjust the treatment according to the results of culture DST testing, once available, } \\
\text { applying a hierachical principle }\end{array}$} \\
\hline \multicolumn{2}{|r|}{$\downarrow$} \\
\hline \multicolumn{2}{|c|}{ Use a hierachical approach to select at least 4 active drugs according DST results of $M$. tuberculosis } \\
\hline \multicolumn{2}{|c|}{$\begin{array}{l}\text { 1. levofloxacin or moxifloxacin } \\
\text { 2. amikacin, kanamycin or capreomycin } \\
\text { 3. prothionamide/ethionamide } \\
\text { 4. cycloserine/terizidone } \\
\text { 5. p-aminosalicylic acid } \\
\text { 6. linezolid } \\
\text { 7. other Group } 5 \text { drugs } \\
\text { (the combination of } 3 \text { and } 5 \text { is not generally recommended) }\end{array}$} \\
\hline $\begin{array}{l}\text { Add ethambutol and pyrazinamide when the rest } \\
\text { count them among the } 4 \text { active drugs }\end{array}$ & of DST suggest that they might be useful, but do not \\
\hline
\end{tabular}

DST result of $M$. tuberculosis from the index patient not known Active TB 


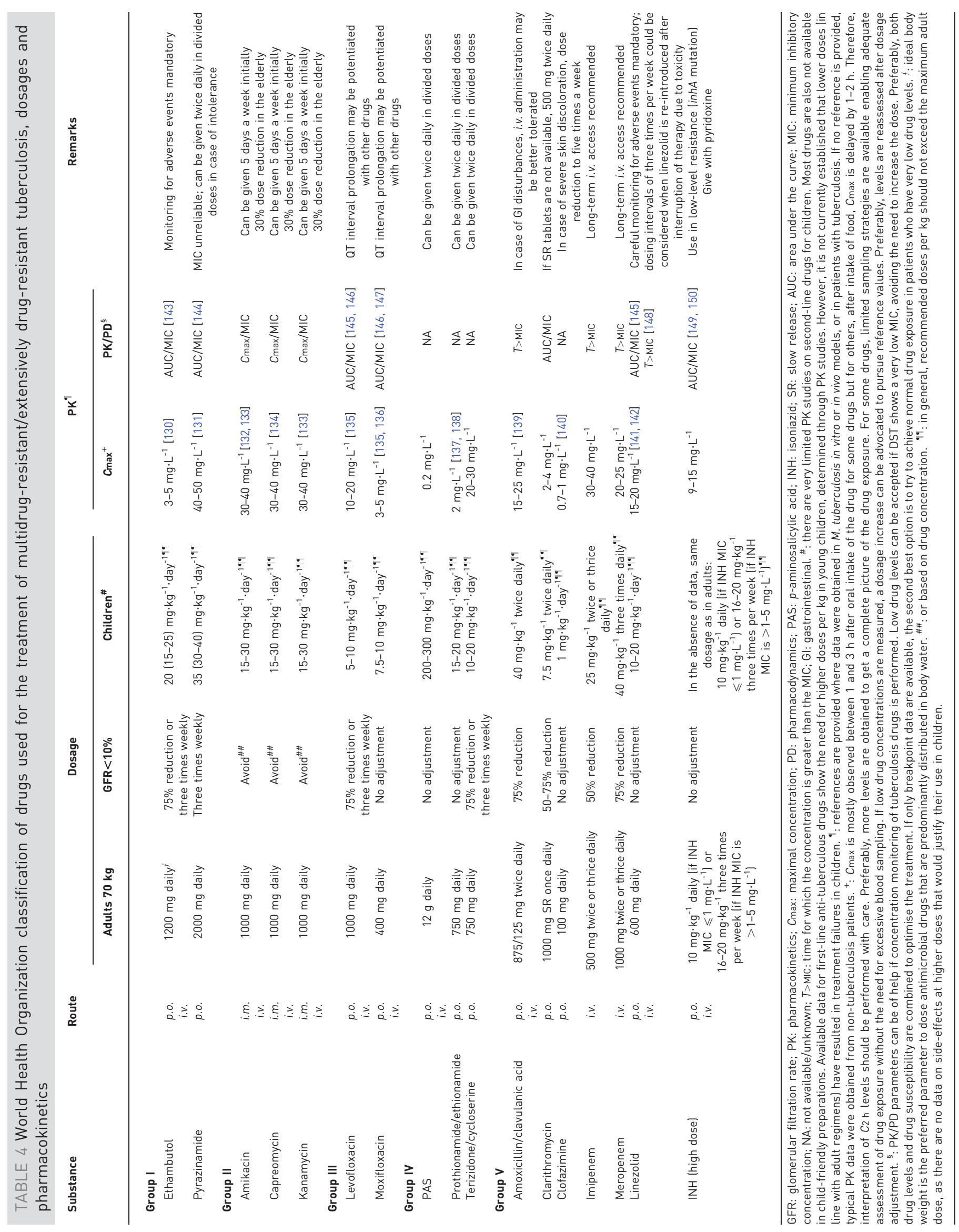


prolonged use) [106, 153], a recent meta-analysis demonstrated that $81.8 \%$ of $121 \mathrm{MDR} / \mathrm{XDR}-\mathrm{TB}$ patients given linezolid-containing regimens were successfully treated [154]. Price is a practical problem that may be partly resolved by using a nonproprietary source [155], provided that drug quality can be assured. Neurotoxicity may be ameliorated by reducing the daily dose [151] or possibly by giving linezolid intermittently [156]. The best dosing schedule of linezolid in the treatment of MDR-TB is still uncertain.

High-dose isoniazid $10 \mathrm{mg} \cdot \mathrm{kg}^{-1} \cdot \mathrm{day}^{-1}$ (when the isoniazid minimum inhibitory concentration (MIC) is $\leqslant 1 \mathrm{mg} \cdot \mathrm{L}^{-1}$ ) or $16-20 \mathrm{mg} \cdot \mathrm{kg}^{-1}$ three times per week (when isoniazid MIC is $>1$ to $5 \mathrm{mg} \cdot \mathrm{L}^{-1}$ ) is possibly beneficial, subject to tolerance and safety $[149,150]$.

Clofazimine is a fat-soluble riminophenazine dye used in the treatment of leprosy worldwide. Two systematic reviews of clofazimine for the treatment of drug-resistant TB have been published [140, 157]. One suggested favourable outcomes (cure or treatment completion) among an overall 65\% (95\% CI $54-76 \%$ ) of drug-resistant TB patients given clofazimine, with little difference between those with MDR-TB (65\%, 95\% CI 52-79\%) and XDR-TB (66\%, 95\% CI 42-89\%) [140]. Although clofazimine has been considered useful in the treatment of XDR-TB [158], one systematic review suggested that, subject to possible selective bias, clofazimine might not add much to a linezolid-based regimen [152].

Recently, a combination of meropenem/clavulanic acid was explored for the treatment of patients with MDR/XDR-TB. In a case-control study of patients with MDR/XDR-TB, a regimen including meropenem/ clavulanic acid plus linezolid was reported to be associated with a smear conversion rate at 3 months of $87.5 \%$ versus $56.3 \%(\mathrm{p}=0.02)$ in controls [159].

\subsection{Ideal treatment regimen for MDR/XDR-TB}

Due to the complexity of the decisions involved, the choice of a MDR-TB treatment regimen is preferably formulated under the guidance of experienced clinicians with due reference to internationally accepted guidelines $[10,25,120,126]$. In several countries, secure websites have been established and hosted by experienced healthcare professionals to discuss treatment decisions and the clinical course of MDR/XDRTB patients with "consilia", face-to-face or via electronic communication [153, 160].

Based on clinical evidence from non-XDR MDR-TB patients, WHO has recommended a standardised MDR$\mathrm{TB}$ treatment regimen that consists of pyrazinamide plus four drugs, namely a fluoroquinolone (preferably a later-generation drug such as moxifloxacin or levofloxacin, avoiding ciprofloxacin), a SLID, a thionamide (ethionamide or prothionamide), and one more oral bacteriostatic drug (preferably cycloserine) [120, 126, $128,151,161,162]$. WHO has recommended use of pyrazinamide (conditional recommendation, very lowquality evidence) without pyrazinamide susceptibility testing, which is technically difficult and often not performed. It is unclear whether this recommendation should apply to all patients, especially when hepatotoxicity is anticipated in patients with underlying chronic viral hepatitis. To facilitate formulation of second-line treatment regimens, patients with MDR/XDR-TB can be classified into two subgroups: 1) new patients with a contact history of MDR/XDR-TB; and 2) new patients without known history of contact with patients with MDR/XDR-TB or patients with previous MDR/XDR-TB treatment.

New patients with a contact history of MDR/XDR-TB

With a contact history of MDR/XDR-TB, such patients should initially receive the same regimen as the putative index case $[23,28,124]$, with possible individualisation according to the index's DST results.

\section{New patients without known history of contact with patients with MDR/XDR-TB or patients with previous MDR/XDR-TB treatment}

In Europe, these patients should have fast access to a rapid genetic DST including rifampicin, isoniazid, fluoroquinolones and SLIDs (in spite of unsatisfactory predictive value for these two last groups of drugs), and the results discussed in a consilium. However, in the settings where these rapid genetic DST is not available, while waiting for DST results, these patients should initially receive the WHO standardised recommended SLD regimen. In MDR-TB high-prevalence settings, it may be necessary to modify the WHO MDR-TB standardised regimen according to the local drug-resistance pattern, especially when the prevalence of bacillary resistance to fluoroquinolones/SLIDs is high. As soon as the results from the culture DST are available, the treatment regimen should be individualised according to the DST result to fluoroquinolones and SLIDs (the DST to the other SLD should be carefully evaluated because the clinical reliability of this DST is very poor). We agree with the WHO recommendation that the regimen should consist of at least four drugs that are likely to be effective, choosing the drugs in a hierarchical order according to their expected activity. It is widely agreed that ethambutol and pyrazinamide should be included in the MDR/XDR-TB drug regimen, when the results of DST suggest their use for the treatment. However, these drugs should not be counted among the four active drugs. For the other drugs, the order 
fluoroquinolone, SLID, prothionamide/ethionamide, cycloserine/terizidone, $p$-aminosalicylic acid (PAS), linezolid then other group 5 drugs is considered to be reasonable, although studies comparing the effectiveness of different regimens are lacking. Where available, linezolid is used by many expert physicians as the first option of group 5 drugs. Most experts in this document agree that clofazimine and meropenem/ clavulanic acid may be more effective for the treatment of XDR-TB than clarithromycin or amoxicillin/ clavulanic acid, although again evidence is lacking. Treatment with high-dose isoniazid should be considered when the drug resistance is related to a mutation in the inhA gene of M. tuberculosis.

All TB patients, including those with XDR-TB, have a chance of being cured [18]. The chance of cure increases with rapid diagnosis and prompt treatment using effective multidrug regimens in a wellfunctioning TB programme that is built on the DOTS strategy (direct observation, short course) and drugresistance treatment programmes $[120,161,163]$. Where available, drug concentration monitoring can be used to optimise drug exposure, thereby contributing to effective and safe TB treatment (table 4).

\subsection{New drugs}

New drugs that have been evaluated for the treatment of TB in clinical phase II-III include linezolid, bedaquiline (TMC207), delamanid (OPC-67683) and PA-824 [162, 164, 165]. Of the new compounds, delamanid and PA-824 are two nitroimidazopyrans with encouraging trial results to date [166-169]. Delamanid promised a good early bactericidal activity in phase I studies [167]. It also demonstrated efficacy as an add-on to a MDR-TB backbone treatment regimen in one recently published randomised, placebocontrolled phase IIb study [166]. On November 22, 2013, the EMA recommended conditional authorisation of delamanid (Deltyba; Otsuka Novel Products, Wexham, UK) for use in combination with other medicines against MDR/XDR-TB, when an effective treatment regimen cannot otherwise be devised for reasons of resistance or tolerability [170]. Due to the limited data available on the efficacy and effectiveness of delamanid for the treatment of MDR/XDR-TB at present, the EMA concluded that additional studies on the long-term effectiveness of delamanid should be conducted [170].

Another promising compound in clinical phase IIb is the diarylquinoline bedaquiline, which inhibits the proton pump ATP synthase of M. tuberculosis. Bedaquiline is active against drug-sensitive and drugresistant strains of $M$. tuberculosis in pre-clinical evaluations. The published results of a phase IIb clinical trial with bedaquiline as an add-on to a MDR-TB backbone treatment regimen showed significant improvement in the rate of culture conversion by 2 months [171]. Since December 31, 2012, bedaquiline has been approved for use in the USA. On December 19, 2013, the EMA has adopted a positive opinion, recommending granting a conditional marketing authorisation for bedaquiline (Sirturo; Janssen Therapeutics, Titusville, NJ, USA) for the treatment of MDR/XDR-TB.

With the availability of new drugs for the treatment of MDR/XDR-TB, the hierarchical order for choosing anti-TB drugs for a MDR/XDR-TB treatment regimen will have to be redefined and updated regularly.

At present, the optimal treatment indication and duration of therapy with delamanid or bedaquiline is unclear. It is also unclear whether it is sufficiently safe to use delamanid and bedaquiline together (or, for example, in combination with moxifloxacin and/or clofazimine) in the treatment of MDR/XDR-TB as all of these drugs may lead to an increase in the QT interval with the potential risk of fatal cardiac arrhythmias. While physicians need to be aware of possible potentiating adverse events with new drugs in a combination regimen and are advised to monitor patients closely, new drugs should be available for all patients who would benefit from them in view of the overall limited prognosis in MDR/XDR-TB.

\subsection{Surgery in the treatment of MDR/XDR-TB}

In the great majority of patients with pulmonary $\mathrm{TB}$, surgery is not indicated. Lung resection should be considered if drug therapy per se cannot ensure cure [121] and when the following criteria are all met: a fairly localised lesion with an adequate postoperative respiratory reserve [120] and effective drugs are available to facilitate postoperative healing of the bronchial stump [122, 172]. Thus, adjunctive surgery may be justifiable in carefully selected patients with XDR-TB/fluoroquinolone-resistant MDR-TB. It cannot be overemphasised that surgery should be performed by experts with support from intensive care units to reduce perioperative morbidity and mortality $[23,153,173]$.

\subsection{Conclusions}

1) The majority of patients with MDR-TB can be cured. With the emergence of fluoroquinolone-resistant MDR-TB and XDR-TB, treatment has become more difficult and costly, with favourable outcomes rarely exceeding 50-60\%. Development of suitable tests for early diagnosis and availability of appropriate treatment are urgently needed to improve the treatment of MDR/XDR-TB. 
2) Patients with XDR-TB also have a chance of being cured, although treatment success is lower than in MDR-TB. The chance of cure increases with rapid diagnosis and prompt treatment using effective multidrug regimens in a well-functioning TB programme that is built on the DOTS strategy and drugresistance programmes.

3) Treatment of MDR/XDR-TB can be individualised according to contact history, drug history and DST.

4) At least four TB drugs that are known or likely to be effective should be given in an MDR/XDR-TB regimen. Adding pyrazinamide to the treatment regimen has been advocated. However, it is unclear whether this recommendation should apply to all patients. Drugs not previously used by the patient or those with in vitro activity based on reliable DST results are likely to be effective.

5) The selection of effective TB drugs to be included in a MDR/XDR-TB regimen should follow a hierarchy of drug groups (fig. 2 and table 4).

6) In MDR-TB high-prevalence settings, it may be necessary to modify the standard WHO MDR-TB treatment regimen according to the local drug-resistance pattern, especially when the prevalence of bacillary resistance to fluoroquinolones/SLID is high.

\section{Duration of MDR/XDR-TB treatment}

One of the most difficult current controversies in the treatment of MDR/XDR-TB is the optimal duration of therapy [124]. Neither the optimal duration of the initial intensive phase, when an injectable agent such as amikacin, capreomycin or kanamycin is usually given, nor the total duration of therapy have been tested in a randomised trial. Prior to 1970, standard therapy for drug-sensitive TB was $\geqslant 18$ months with three or more drugs $[174,175]$. Subsequent to the introduction of rifampicin into clinical practice in 1970, multiple randomised trials were conducted, which demonstrated that, with use of rifampicin, the total duration of therapy could be reduced to 9 months $[176,177]$. Subsequent trials demonstrated that, with the addition of pyrazinamide, total therapy duration could be further reduced to 6 months $[176,177]$. Given the historic experience with regimens without rifampicin, it has been conventional wisdom that 18 months should be the minimum total duration of therapy for patients with TB caused by rifampicin-resistant bacteria.

Recently, individual patient records from 32 centres that had reported the results from cohorts of patients were assembled into a single pooled data set. Most of these received individualised (and nonrandomised) treatment. These data comprised a total of 9898 patients with MDR-TB of whom 9153 had pulmonary MDR-TB [178]. The availability of detailed individual patient data enabled analysis of treatment covariates with stratification, restriction or adjustment for a number of confounding variables such as age, previous treatment, drug-resistance pattern or HIV infection. Analysis of this large data set revealed that the optimal duration of the initial intensive phase was 7-8.4 months and the optimal total duration of therapy was 19-21 months. These findings are summarised in figure 3. A number of additional sensitivity analyses were performed, such as restricting to adult patients who had never been treated with SLDs before, excluding patients who were treated only with FLDs or excluding HIV-infected patients [178]. These sensitivity analyses confirmed the findings, which form the basis of recent recommendations by WHO on the management of MDR-TB, which recommend that the duration of treatment is modified according to the response to therapy [126]. Evidence-based recommendations for the duration of treatment for patients with $\mathrm{XDR}-\mathrm{TB}$ are not available. In the absence of evidence, recommendations for the duration of treatment in MDR-TB currently also apply to patients with XDR-TB.

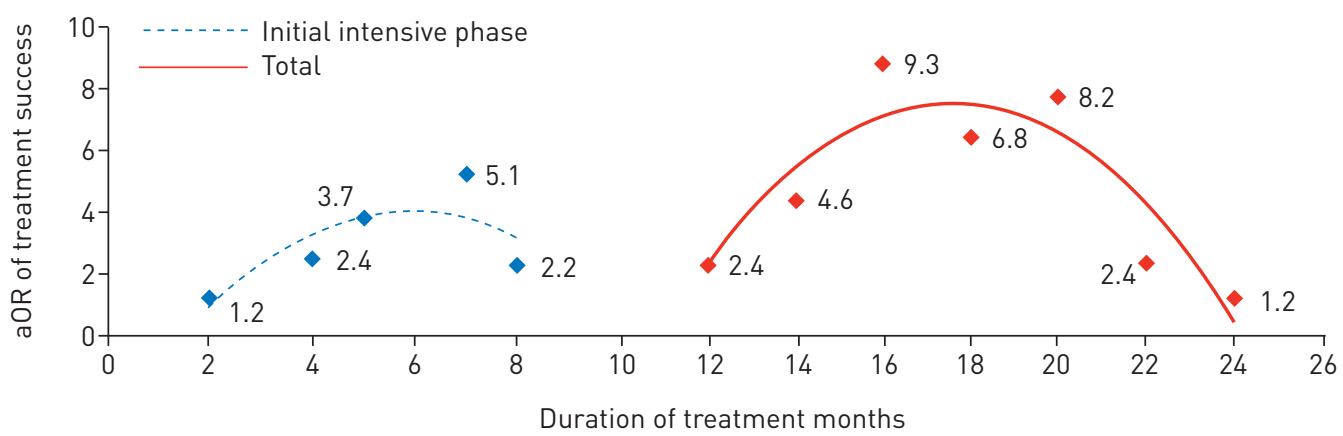

FIGURE 3 Association of the duration of initial intensive phase and total treatment with adjusted odds of treatment success in patients with multidrug-resistant tuberculosis. aOR: adjusted odds ratio. Data from [178]. 
Of course, this long duration creates a number of problems, including a greater risk of toxicity, potentially greater default rates, greater health system costs and greater economic losses for patients who often cannot work. The high costs associated with the treatment of MDR/XDR-TB may represent an intolerable burden to some National TB Programmes [179].

Two drugs that might allow shortening of current MDR/XDR-TB therapy are pyrazinamide and the fluoroquinolones. However, resistance to pyrazinamide is common among patients with MDR/XDR-TB (in the individual patient data meta-analysis, slightly over half of those tested had resistance to pyrazinamide), reflecting prior use in most of these patients [178]. Fortunately, resistance to fluoroquinolones is much less common and the fluoroquinolones are highly active against $M$. tuberculosis, particularly the later generation fluoroquinolones, such as levofloxacin, moxifloxacin or gatifloxacin [180]. A nonrandomised study from Bangladesh reported excellent results with a much shorter regimen, which used gatifloxacin throughout [106]. Of the 9153 MDR-TB patients referred to earlier, only $14 \%$ had access to later generation fluoroquinolones [178]. It is possible that the results would have been different if more centres had these drugs available.

Many experts will recommend shorter regimens for patients with smear-negative or less radiologically extensive disease and patients with little prior exposure to TB treatment, particularly to SLDs, as well as those with smear or culture conversion in $<3$ months. It should be noted that these suggestions are based on expert opinion and personal experience $[25,181]$. WHO has provided guidance to countries listing the criteria under which these shorter regimens for MDR-TB treatment can be used [24]. In order to individualise the duration of therapy for patients with MDR/XDR-TB, identification and validation of biomarkers that indicate a point in time during therapy corresponding to relapse-free treatment success are urgently needed.

A randomised controlled trial (STREAM) is ongoing to test a shorter treatment duration regimen. This trial is expected to end in October 2016 [182]. Results from the STREAM trial will be crucial in determining whether therapy for MDR-TB can be successfully shortened and, if so, in which groups of patients. Treatment with new drugs, like bedaquiline and delamanid, may allow shortening of MDR/XDR-TB therapy, although it is likely that several trials will be required to determine the optimal duration of therapy with these new agents.

\subsection{Conclusions}

1) The optimal duration of the initial phase and total therapy of MDR-TB is controversial, reflecting the limited evidence. There is an urgent need for randomised clinical trials to assess the optimal duration of treatment of MDR/XDR-TB.

2) Biomarkers for the indication of relapse-free treatment success are urgently needed to individualise the duration of MDR/XDR-TB treatment.

3) Based on the analysis of the most comprehensive published evidence currently available, derived from an individual patient data meta-analysis of $>9000$ adult patients with MDR-TB, WHO has recommended treatment duration for patients with MDR-TB for the initial intensive phase of $7-8.5$ months and total treatment of 20 months.

4) In the absence of evidence, recommendations for the duration of treatment of MDR-TB currently also apply to patients with XDR-TB.

5) However, based on expert opinion, there may be situations where shorter regimens, particularly in the initial intensive phase, are appropriate. These include patients with less extensive disease, no prior treatment (especially with SLDs), and rapid conversion of sputum smears and cultures, and possibly children.

\section{Management of adverse drug events in MDR/XDR-TB}

Drugs used to treat MDR/XDR-TB are often associated with adverse effects [66, 183, 184]. These can occur early or late in treatment. It is very important to anticipate the development of adverse events and to inform patients about this. Giving treatment and, in some cases, discontinuing or changing medication can all help improve adherence and enable the patient to complete their course of treatment. Ensuring that the patient understands the importance of each medication, the possible side-effects of medication they are taking and what to do when they occur can reduce the risk of serious adverse events. Herein, we present a brief description of adverse events by system. Table 5 outlines the main adverse effects of each drug class and provides management guidance.

\subsection{Gastrointestinal}

Nausea and vomiting are the most common side-effects [184]. Often, they start a few days after commencing medication. Antiemetics should be offered, when needed. Drugs might be given at a different time or drug 
TABLE 5 Common adverse effects of drugs used for the treatment of multidrug-resistant/extensively drug-resistant tuberculosis and their management

\begin{tabular}{|c|c|c|}
\hline Substance & Common adverse effects & Management \\
\hline \multicolumn{3}{|l|}{ Group I } \\
\hline Ethambutol & Optic neuropathy & $\begin{array}{l}\text { Inform the patient to report decreased vision immediately. Discontinue and refer } \\
\text { to an ophthalmologist if vision deteriorates. More likely to occur in patients with } \\
\text { renal impairment. }\end{array}$ \\
\hline Pyrazinamide & Hepatotoxicity, rash, gout & $\begin{array}{c}\text { For hepatotoxicity, stop the drug; reintroduce in an escalating dose over } \\
\text { several days. Discontinue drug if hepatotoxicity reoccurs. For rash, manage } \\
\text { symptomatically; if extensive, stop drug and consider reintroduction. Discontinue } \\
\text { if rash reoccurs. For gout, reduce dose initially and consider starting allopurinol } \\
\text { when acute attack has settled. }\end{array}$ \\
\hline \multicolumn{3}{|r|}{ - } \\
\hline Amikacin & Ototoxicity, nephrotoxicity & $\begin{array}{l}\text { Monitor levels, hearing and renal function monthly. If problems occur, consider } \\
\text { reducing dose frequency to three times a week. Discontinue if problems persist, } \\
\text { but balance risk of cure versus deafness. }\end{array}$ \\
\hline Capreomycin & Ototoxicity, nephrotoxicity & $\begin{array}{l}\text { Monitor levels, hearing and renal function monthly. If problems occur, consider } \\
\text { reducing dose frequency to three times a week. Discontinue if problems persist, } \\
\text { but balance risk of cure versus deafness. }\end{array}$ \\
\hline Kanamycin & Ototoxicity, nephrotoxicity & $\begin{array}{l}\text { Monitor levels, hearing and renal function monthly. If problems occur, consider } \\
\text { reducing dose frequency to three times a week. Discontinue if problems persist, } \\
\text { but balance risk of cure versus deafness. }\end{array}$ \\
\hline \multicolumn{3}{|r|}{ 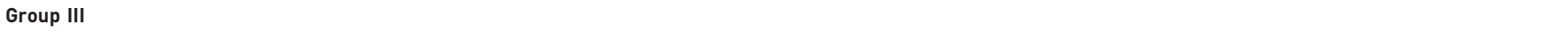 } \\
\hline Levofloxacin & GI disturbances, tendinitis, insomnia & QT interval prolongation may be potentiated with other drugs \\
\hline Moxifloxacin & GI disturbances, tendinitis, insomnia & QT interval prolongation may be potentiated with other drugs \\
\hline \multicolumn{3}{|r|}{ 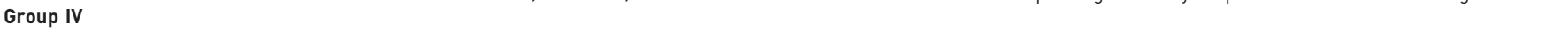 } \\
\hline PAS & $\begin{array}{c}\text { Nausea and vomiting, gastritis, hepatotoxicity, } \\
\text { hypothyroidism }\end{array}$ & $\begin{array}{l}\text { Rehydrate if necessary. Give antiemetics } 30 \text { min before the medication; several } \\
\text { classes of antiemetic may need to be tried. Twice or three times a day divided dose } \\
\text { may help. Gastritis can be helped by administering the drug with a small amount } \\
\text { of food or giving an antacid or } \mathrm{H} 2 \text { blocker. For hypothyroidism, check TFT. }\end{array}$ \\
\hline Prothionamide/ethionamide & $\begin{array}{l}\text { GI disturbances, depression, hepatotoxicity, } \\
\text { hypothyroidism }\end{array}$ & $\begin{array}{c}\text { As above. Depression can be treated with an antidepressant if other } \\
\text { causes excluded. }\end{array}$ \\
\hline Terizidone/cycloserine & Neurotoxicity, peripheral neuropathy & $\begin{array}{l}\text { Give high-dose pyridoxine, up to } 50 \mathrm{mg} \text { for every } 250 \mathrm{mg} \text { of drug. If neuropathy } \\
\text { progresses, discontinue drug. Discontinue if psychosis develops. Seizures can be } \\
\text { managed with anticonvulsants, but drug may need to be discontinued. }\end{array}$ \\
\hline \multicolumn{3}{|r|}{ 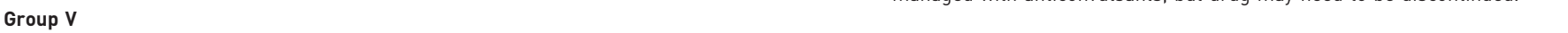 } \\
\hline Amoxicillin/clavulanic acid & Hypersensitivity, GI disturbances & Not suitable for patients with penicillin allergy. \\
\hline Clofazimine & Skin discolouration, $\mathrm{GI}$ disturbances & Inform the patient about discoloration of skin and body fluids \\
\hline Imipenem & Hypersensitivity, neurotoxicity & Monitor blood count \\
\hline Meropenem & Hypersensitivity, neurotoxicity & Monitor blood count \\
\hline Linezolid & Neuropathy, anaemia & $\begin{array}{l}\text { Monitor blood count; avoid prolonged use, when possible. Stop if peripheral } \\
\text { neuropathy or haematological problems occur. Give with pyridoxine. }\end{array}$ \\
\hline Isoniazid (high dose) & Peripheral neuropathy, hepatotoxicity & Give with pyridoxine \\
\hline
\end{tabular}

PAS: $p$-aminosalicylic acid; GI: gastrointestinal; TFT: thyroid function test.

dosages can be split across the day where possible, while other drugs that may cause gastric irritation should be avoided (e.g. nonsteroidal anti-inflammatory drugs (NSAIDs)). Proton pump inhibitors might be considered for gastritis. Hydration should be encouraged. A light snack before the medication may reduce nausea. One practical problem is to decide if the drug dose has to be repeated if the patient has vomited after the intake of the first pills, as the drugs may not have been absorbed. If the patient vomits $<30 \mathrm{~min}$ after taking their dose of medication, they should be advised to take the dose again after a suitable antiemetic.

Diarrhoea can be caused directly by medication, such as PAS, although other causes should be excluded. Stools should be checked for Clostridium difficile, especially in patients taking fluoroquinolones.

Hepatotoxicity should be suspected in any patient with gastrointestinal disturbance and liver enzymes must be checked at regular intervals [185] (table 6). If the transaminases are raised by more than three times the upper limit of normal, hepatotoxic medication should be suspended until they normalise. For smaller rises in transaminases, if the patient is well, it is advised to monitor liver enzymes weekly. Other causes of hepatotoxicity, such as viral hepatitis, other concomitantly prescribed medication and alcohol, should always be excluded.

\subsection{Dermatological}

Rashes and itching are common side-effects early in MDR-TB treatment [186]. If they are mild, treatment can be continued and symptomatic relief with antihistamines and hydrocortisone cream can be offered. Other causes of rash and itching, such as scabies, eczema, psoriasis, tinea or liver disease, need to be excluded. If there is any systemic upset, such as fever, urticaria, blistering or mucus membrane involvement, 


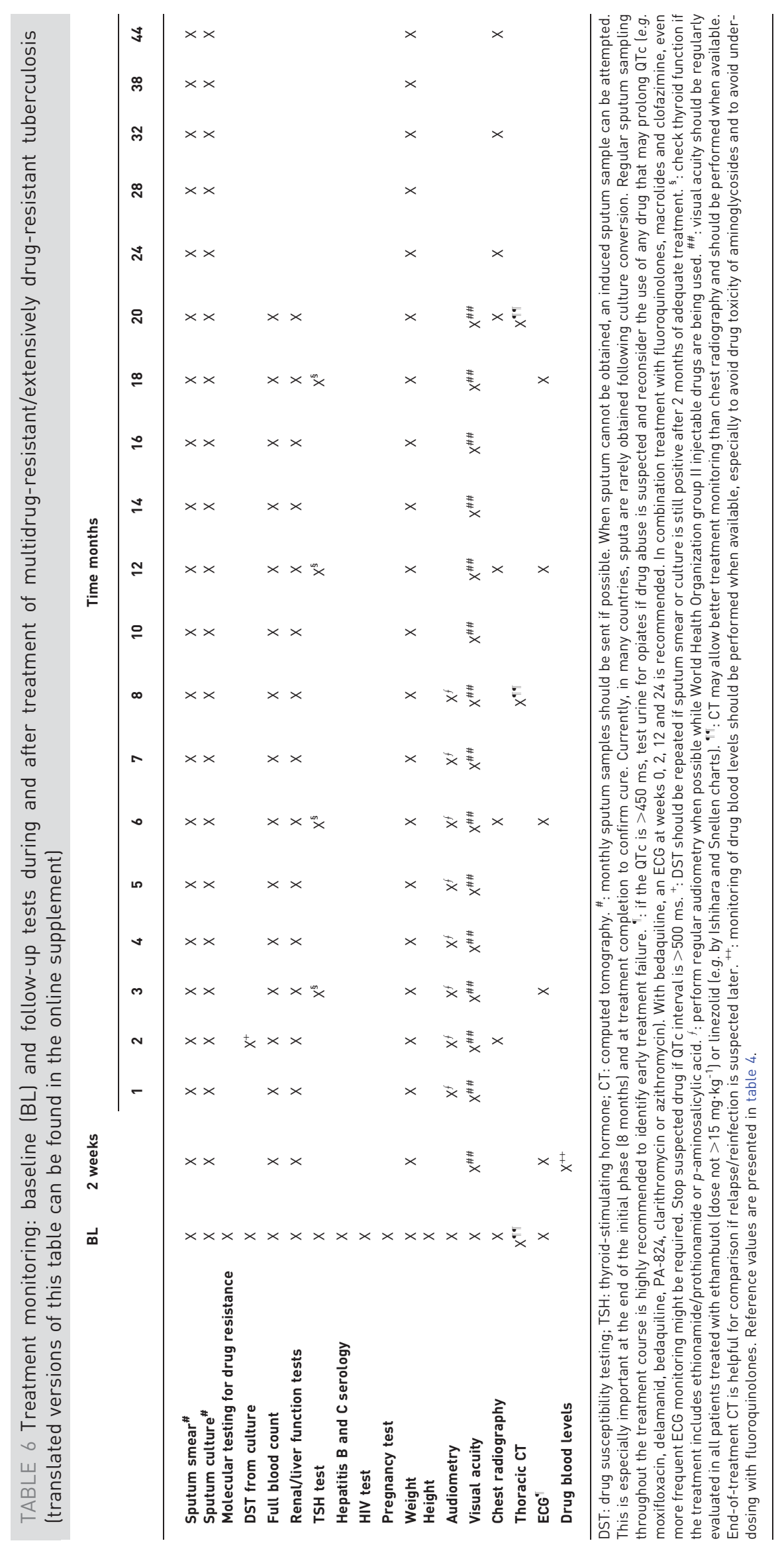


treatment must be discontinued immediately. For nonanaphylactic drug reactions, medication can often be reintroduced in a gradual fashion.

\subsection{Psychoneurological}

Peripheral neuropathy is more common in patients with diabetes, excess alcohol use, malnutrition and HIV infection. Pyridoxine should always be added when treatment includes high-dose isoniazid, cycloserine, thionamides or linezolid. Central nervous system toxicity ranges from headaches to seizures and psychosis [187]. Other causes that affect central nervous system function should always be excluded before discontinuing medication. Drug-specific adverse effects are shown in table 5. Most commonly, ototoxicity occurs during treatment with aminoglycosides/polypeptides and patients should be warned about hearing or balance changes [188]. In this case, the medication must be discontinued immediately. Monthly audiograms are advisable. Optic neuritis can occur as a complication of treatment with ethambutol or linezolid $[189,190]$. Treatment with these drugs should be discontinued in the event of visual loss. Monthly examination of visual accuracy, especially red-green discrimination, is suggested.

\subsection{Renal}

Renal toxicity is usually due to aminoglycosides or capreomycin [188]. Careful monitoring of renal function during treatment and measurement of aminoglycoside levels can minimise problems. Other nephrotoxic drugs, such as NSAIDs, should be avoided in this situation where possible.

\subsection{Haematological}

Several drugs cause anaemia, leukopenia and thrombocytopenia. Other causes of haematological abnormalities should be excluded before stopping TB medication.

\subsection{Miscellaneous}

Hypothyroidism can occur with either PAS or ethionamide/prothionamide [191]. Baseline thyroidstimulating hormone (TSH) should be assessed and monitored 3-monthly. Thyroxine should be replaced when TSH rises above twice normal. Gynaecomastia, metallic taste in the mouth and mild alopecia have all been reported with ethionamide. These side-effects can usually be managed with reassurance alone. Rupture of the Achilles tendon may occur in long-term treatment with fluoroquinolones.

\subsection{Conclusions}

1) It is important to counsel patients on possible adverse drug events so they know what to expect and can report them early.

2) Many drug-related adverse events can be prevented by careful monitoring of drug dosage, and anticipation and treatment of side-effects.

3) For nonanaphylactic drug reactions, medication can often be reintroduced in a gradual fashion.

\section{Special considerations for MDR/XDR-TB in-patient case management}

It is agreed that the initial management of patients with MDR/XDR-TB in Europe should generally take place in a hospital setting. All patients suspected of having pulmonary MDR/XDR-TB should be individually isolated after admission to a hospital to avoid nosocomial transmission of $M$. tuberculosis (see chapter 7 for infection control measures). Patients need to be informed about TB, preventive measures, diagnostic procedures and treatment modalities in a language that they understand. Brochures and videos (www.explaintb.org) can be very helpful for patient education and to promote adherence. When the diagnosis of MDR/XDR-TB is confirmed, the long-anticipated treatment duration and related issues of isolation and infection control and adherence need to be discussed with the patient. In the great majority of low TB incidence countries in Europe, patients with MDR/XDR-TB often have a background of recent migration [192, 193]. In fact, MDR/XDR-TB is sometimes the reason for healthcare-seeking migration. Psychosocial support is particularly important in patients who lack local social networks including recent visits from family and friends.

Rapid molecular resistance testing should be the standard of care for all patients evaluated for MDR/XDRTB $[26,66]$. Treatment should ideally be initiated when molecular test results are available. The cost of molecular testing, available at national and supranational reference laboratories throughout Europe, is less than the cost to the patient and to a health system of an inadequate regimen.

Unless a patient is very ill, treatment initiation is not an emergency. However, the risk of nosocomial transmission decreases substantially as soon as a patient receives adequate treatment and treatment should not be unnecessarily deferred. Comorbidities, such as HIV infection (see chapter 16), viral hepatitis, 
diabetes mellitus and potential drug-drug interactions, need to be thoroughly evaluated before anti-TB therapy is started. The decision to initiate anti-TB therapy and the choice of the combination drug regimen should be made by a physician with experience in the care of patients with MDR/XDR-TB, preferably in agreement with a consilium. When patients are initially admitted to nonspecialised departments, transfer to a specialised referral centre is to be preferred. This is especially important in low TB incidence countries in western Europe, where experience with complicated cases of TB is usually restricted to very few centres in a country.

Prior to the initiation of therapy, a set of baseline investigations is required (table 6). When the choice of a drug regimen is made, all drugs should be administered at once without stepwise introduction. Directly observed treatment should be ensured in the in-patient setting. Drug concentration measurement can be used to optimise drug exposure.

When patients receive injectable drugs, implantation of a peripherally inserted central catheter (PICC line) or, preferably, a central venous catheter with a subcutaneous silicon reservoir for injections (e.g. PORT-ACATH; Smiths Medical, St Paul, MN, USA) should be encouraged to avoid daily intramuscular or intravenous injections through peripheral veins over a period of several months.

Depending on the treatment regimen, patients should be informed about signs and symptoms of adverse drug events. Patients need to know that the medication must be discontinued immediately under certain circumstances, such as when hearing or balance impairment develops.

Patients who smoke cigarettes should be strongly encouraged to stop smoking as it improves the chance of a positive treatment outcome [194]; nicotine replacement therapy should be offered.

Continued alcohol intake may lead to hepatotoxicity, which can be difficult to distinguish from drug toxicity. Abstinence from alcohol is recommended and treatment with benzodiazepines and thiamine should be given to cover withdrawal. Opioid substance abuse should be treated with methadone in a substitution programme. The problem of substance abuse should be re-addressed repeatedly and support must be offered to maximise treatment success.

Malnutrition is common. In addition to a good diet, vitamin D may improve sputum smear conversion in patients, depending on a vitamin D receptor polymorphism [195]. A diet rich in cholesterol has been associated with accelerated sputum conversion [196]. In severely malnourished individuals, enteric feeding should be considered if providing high-caloric drinks does not result in weight gain. Feeding via a percutaneous endoscopic gastrostomy may be required for those who cannot take regular meals (both methods can deliver drugs whose taste affects adherence). Vitamin $\mathrm{B}_{6}\left(50 \mathrm{mg} \cdot \mathrm{day}^{-1}\right.$ for adults, optimal dosage unknown) should be given with high-dose isoniazid and cycloserine, and has also been suggested for linezolid and the thionamides [197].

Physiotherapy and occupational therapy are often withheld from in-patients with TB, especially patients with MDR/XDR-TB, because of the risk of infection for the hospital staff. Due to the long duration of inpatient treatment for those with MDR/XDR-TB [198], regular exercises for body and mind are especially important for these patients. Hospitals must provide possibilities for regular physical activities (e.g. exercise bikes in isolation rooms and open-air sports) to ensure muscle mass is regained with treatment. Occupational therapy and the use of the new social media can reduce the sense of isolation and stimulate the mind. When patients are unable to communicate in the language of the hospital staff, regular conversations with interpreters should be arranged. Face-to-face interpretation is preferred. Sometimes telephone conferences with an interpreter may be helpful to communicate with a patient.

Response to treatment and adverse drug events should be assessed and monitored at regular intervals (table 6). Sputum smear and culture examination should be performed at least once a month [199]. Time to culture positivity is a valuable measure of response to treatment [200]. In contrast, clinical and radiological scores are poorly validated.

Although patients with MDR/XDR-TB are sometimes discharged from the hospital as early as 2 weeks after initiation of an adequate treatment regimen [74, 79-81], in many centres, patients with MDR/XDR-TB are considered for discharge when three sputum cultures collected over a 14-day period are culture-negative. In practice, some patients may stay in isolation for $>6$ months.

Prior to the discharge of a patient with MDR/XDR-TB from the hospital, continuation of care and monitoring during the outpatient phase of treatment must be ensured [66]. Table 7 provides a practical checklist of issues that need to be addressed and solved before a hospitalised patient with MDR/XDR-TB should be discharged. This checklist is also available as a form for clinical use in the online supplementary material (table S1). 
TABLE 7 Discharge checklist for the end of the in-patient treatment of multidrug-resistant (MDR)/extensively drug-resistant (XDR) tuberculosis (TB) (translated versions of this table can be found in the online supplement)

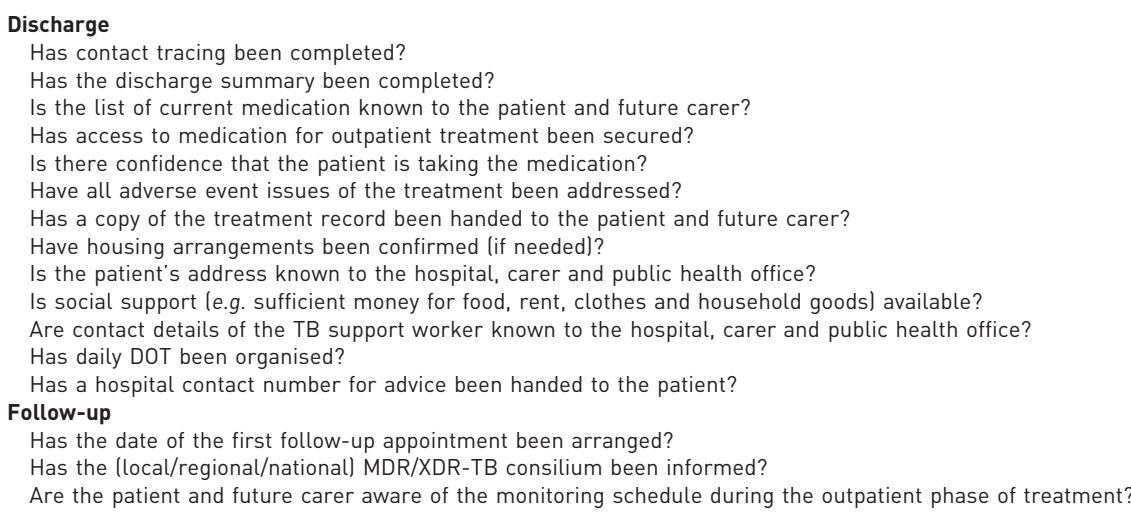

This checklist is also available as a form for use in clinical practice in the online supplementary material (table S1). DOT: directly observed therapy.

\subsection{Conclusions}

1) Patients suspected of having MDR/XDR-TB should be isolated to avoid transmission of M. tuberculosis.

2) Rapid molecular resistance testing, including LPAs for SLD resistance testing, should be the standard of care for all patients evaluated for MDR/XDR-TB.

3) The decision on treatment initiation and the choice of drugs should be made by a physician with experience in the care of patients with MDR/XDR-TB. Transfer of a patient to a specialised treatment centre should be strongly considered.

4) Patients need to be informed about $T B$, preventive measures, diagnostic procedures and treatment modalities in a language that they understand. Brochures and videos can be very helpful for patient education and to promote compliance.

5) Comorbidities, smoking and substances abuse need to be addressed.

6) Adequate nutrition, supplements (vitamin $\mathrm{B}_{6}$ etc.), physiotherapy and psychosocial support should be provided.

7) Response to treatment and adverse drug events should be assessed and monitored at regular intervals.

8) Prior to discharge from the hospital, continuation of care and monitoring during the outpatient phase of treatment must be ensured.

\section{Special considerations for MDR/XDR-TB outpatient case management}

Outpatient treatment of patients with MDR/XDR-TB has been considered problematic because of the need for daily directly observed therapy (DOT), particularly if treatment includes multiple daily doses of medications (including i.v. or i.m. administered medications). Nonetheless, several reports of outpatient treatment of patients with MDR/XDR-TB have demonstrated encouraging results [201-204]. WHO now recommends that patients with MDR-TB should be treated using mainly ambulatory care [66]. In some countries where in-patient capacity is low at the beginning of the treatment, isolation may be required in the patient's own home with the "hospital at home" approach that was employed in the early treatment of drug-sensitive TB [205].

Each patient should have a specifically assigned healthcare worker, so that there is a single point of contact (e.g. for adverse effects, loss of tablets and change in social circumstances). Training of assigned healthcare workers is essential in order to coordinate care across community, hospital and public health services. Outpatient care and the provision of DOT are managed by healthcare providers in a different manner in, and even within, each European country. Coordination, the timely transfer of information by the assigned healthcare worker and a record of each dose of each drug that has been administered are important to ensure that treatment is successfully completed. The response to treatment should be assessed by monthly sputum analysis (including recording of the times of sputum smear and culture conversion), ideally until the end of treatment. However, most patients are unable to provide sputum samples shortly after sputum culture conversion. The inability to produce sputum for microbiological examinations should then be 
recorded. Repeated DST is required if a patient who had a negative sputum smear develops positive sputum smears again or if negative cultures become positive again.

Recommendations for monitoring treatment response (smear, culture and imaging studies), screening for adverse events (audiogram and laboratory tests) and infection control precautions should follow a similar pattern proposed for the in-patient care (table 6).

Great care should be taken to ensure that patients continue to take all their treatment for the duration prescribed. The full participation of the patient is helped by a good relationship with a key worker and by an understanding of the illness [206]. Patient and family education is often needed in order to reduce stigma. Patients must be continuously educated about infection control measures, the importance of ventilation, the need for separate sleeping rooms, and the importance of avoiding exposure to young children and immunocompromised individuals [207, 208]. Incentives, such as transportation subsidy or food, have been shown to increase adherence to treatment, particularly in high-risk groups such as drug users, the homeless and prison inmates [209].

A flexible, organised outpatient centre on the model of the One-Stop TB Shop [210] should be available to manage the cases, provide expert medical advice and deal with adverse events. Regular (e.g. 3-monthly) review of patients with MDR/XDR-TB by a multidisciplinary team (consilium) is helpful to ensure good treatment outcomes (see chapter 18).

Kept hospital appointments are a marker for adherence and follow-up by clinical assessment, and chest radiography is valuable as an early pointer to treatment failure [211]. Identification of potential causes of nonadherence should be sought and resolved.

Facilities for MDR/XDR-TB outpatients should also be available for contact investigations. In order to prevent loss of information between different health services, including the laboratory, and to allow comparability of data, there is a need to define early the information required, harmonising support, responsibilities and flow of information. At the last outpatient visit, treatment outcome, based on standardised definitions, must be recorded, and previous healthcare providers (hospital, consilium, etc.) and surveillance systems must be informed. End-of-treatment imaging studies are very helpful as patients may be reassessed later for suspected relapse or reinfection. Patients must have clear information about signs and symptoms of recurrent TB. They should already have the date of their first follow-up appointment and should be actively followed for $\geqslant 2$ years. The end-of-treatment checklist is presented in table 8 and is also available as a form for clinical use in the online supplementary material (table S2).

\subsection{Conclusions}

1) Each patient with MDR/XDR-TB should have a key worker during the outpatient phase of treatment as single point of contact.

2) Continuous access to medication and medical care must be ensured throughout the outpatient phase of treatment. DOT is advised throughout treatment of MDR/XDR-TB.

3) Response to treatment should be monitored and assessed by regular examinations at standardised intervals, including scheduled visits to a specialised MDR/XDR-TB outpatient department.

4) Identification of potential causes of noncompliance and adverse drug reactions should be sought and resolved.

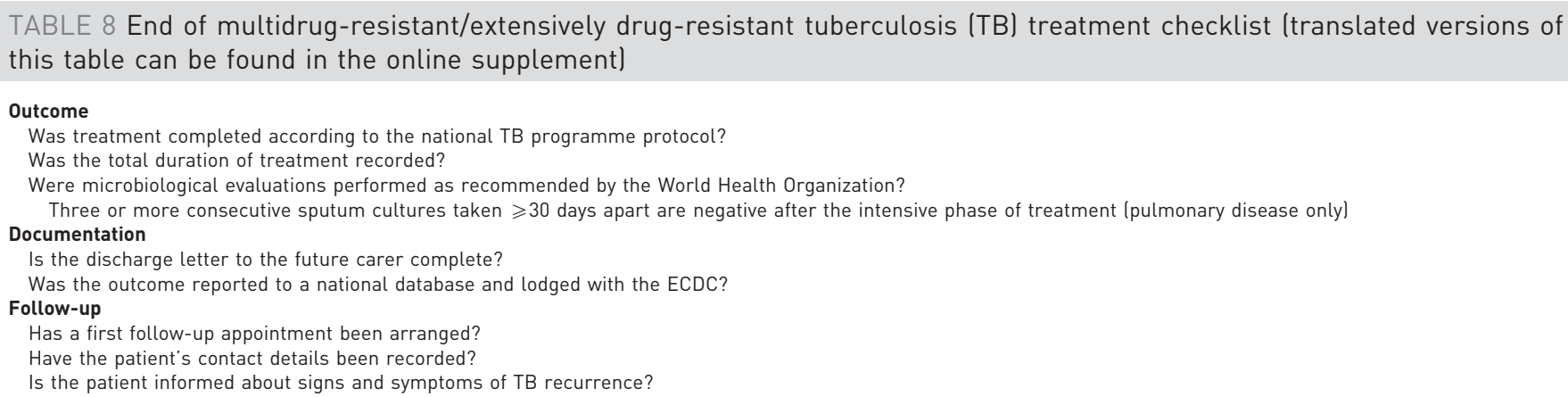

This checklist is also available as a form for use in clinical practice in the online supplementary material (table S2). 
5) At the end of treatment, information should be provided to previous healthcare providers, and registries and patients must be informed about follow-up visits.

\section{Special considerations for MDR/XDR-TB in children}

The well-described limitations surrounding correct and timely diagnosis of drug-sensitive childhood TB apply equally to MDR/XDR-TB: the paucibacillary nature of the disease implies that in the best case scenario, only $30-40 \%$ of children diagnosed with TB have testable isolates in culture systems, depending on age and site of disease [212-215]. In the absence of bacteriological confirmation, children are often not included in global surveys of MDR/XDR-TB incidence, and data on the global burden $[32,216,217]$ and cohort outcome [218-223] are sparse. In addition, the treatment regimens are deduced from the adult recommendations and information on drug pharmacokinetics; safety and dosing data of paediatric MDR/ XDR-TB are currently lacking.

Judging by adult MDR/XDR-TB prevalence figures, it is likely that MDR/XDR-TB in children is seriously under-reported. TB in children is usually transmitted from a sputum smear-positive adult index case and the same applies to MDR/XDR strains [224, 225]. However, rather than developing primary resistance through incomplete or inadequate regimens, as seen in adults, MDR/XDR-TB in children is usually caused by the acquisition of an already-resistant strain [226]. In line with drug-susceptible TB, therefore, a case of MDR/XDR-TB in a child equally represents a sentinel event of transmission in the community and can serve as an indicator for TB control programmes.

The main challenges are not only the management of active cases (confirmed or suspected) but also the uncertainty of what to do with children who have been exposed but do not yet have signs of active disease. We do not know for certain if they require prophylactic treatment to prevent infection or treatment of LTBI with a presumed drug-resistant strain, or if the risks of potential side-effects and use of complicated drug regimens outweigh the benefits.

It is well established that the likelihood of progression to active disease varies with age in drug-susceptible disease and there is no reason to assume that this should be any different for MDR/XDR cases of TB. Hence, management of LTBI and MDR/XDR exposure needs to take age and other risk factors into account. The complexity of these issues has resulted in a dearth of evidence-based guidelines for paediatric MDR/XDRTB. Here, we summarise the available evidence to date and outline the further research priorities.

\subsection{Management of active MDR/XDR-TB in children}

A recent systematic review and meta-analysis of treatment outcomes for children with MDR-TB [227] identified eight studies with reported treatment outcomes in 315 patients. The pooled treatment success, defined as a "composite of cure and treatment completion as defined by the studies" was $81.7 \%$, with a mortality of 5.9\%. 6.2\% defaulted treatment and 39\% had adverse drug events. The use of injectable drugs was associated with better patient outcomes but it was impossible to judge optimal treatment duration.

The available guidelines addressing management of MDR/XDR-TB in children specifically recommend treatment according to the susceptibility of the index case strain if it is not possible to obtain a bacteriological specimen from the child $[228,229]$. A SLID, a fluoroquinolone and at least two other drugs to which the index case strain or the strain isolated from the child is sensitive by DST should be included in the initial drug regimen. Pyrazinamide should be added to these four drugs for the whole course of treatment but should not be counted among the active drugs, providing that DST suggests susceptibility of the causative M. tuberculosis strain to pyrazinamide. Parenteral treatment with the SLID should be continued for at least 4-6 months, or sometimes longer, depending on disease severity. When treatment with the SLID is discontinued, treatment should be continued per os with at least three active drugs, not counting pyrazinamide [229]. The optimal duration of MDR/XDR-TB treatment in children is unclear and may be very variable from case to case. Depending on the extent of the disease, the DST pattern of the causative bacteria and the immune status of the child, a total duration of treatment between 12 and 18 months following culture conversion could be reasonable and treatment may have to be prolonged in some cases to avoid the risk of relapse. WHO guidelines [10] are largely similar, but do not explicitly address children and do not mention DOT. Finally, the decision on the exact number of drugs and length of therapy also depend on extent and site of disease, penetration of the chosen drugs and treatment response. It could be argued that as paediatric TB is usually paucibacillary, bacterial clearance may be achieved more rapidly in children than in adults and, thus, shorter regimens may suffice. LEUNG et al. [181] have described successful reduction of treatment duration for selected adult patients with MDR-TB. However, there are no clinical trials in children supporting this approach at present. 


\subsection{Conclusions}

1) The lack of evidence-based advice for managing paediatric MDR/XDR-TB is internationally acknowledged [10] and significant heterogeneity exists in clinical management, particularly in patients exposed to infectious MDR/XDR-TB. Currently, each case will need to be assessed on an individual basis. Involvement of a childhood $\mathrm{TB}$ expert and adult $\mathrm{TB}$ colleagues in these challenging situations is recommended.

2) In the absence of a bacterial isolate, the regimen should be designed around the sensitivities of the index cases strain.

\section{Special considerations for MDR/XDR-TB in pregnancy}

There is consensus that neither LTBI following contact of a patient with MDR/XDR-TB nor active MDR/ XDR-TB during pregnancy requires termination of pregnancy [230]. While treatment of females who develop MDR/XDR-TB during pregnancy or become pregnant during treatment can be successful without adverse events for the newborn [230-233], the safety of many drugs for the unborn child is unknown. FLDs are not considered teratogenic but there is little knowledge about human use of SLDs during pregnancy. A case series of treatment of pregnant females with amikacin, ofloxacin, prothionamide, cycloserine and pyrazinamide did not show any harm to the children or their mothers [233]. In contrast, streptomycin and kanamycin caused ototoxicity in the children [234]. Hence, these drugs and other aminoglycosides/ polypeptides are not recommended for treatment during pregnancy. Fluoroquinolones have caused arthropathy in animals but have been safely used in pregnant women [235]. Ethionamide has been implicated in congenital defects in animals [236], although human data are not available [232].

Breastfeeding should be recommended only in females who are not infectious. In general, although drugs are found in the breast milk, the benefits of breastfeeding of children with mothers on MDR/XDR-TB treatment outweigh the potential harm related to ingestion of TB drugs through breastfeeding [237].

\subsection{Conclusions}

1) Safe treatment of MDR/XDR-TB during pregnancy seems possible but needs individual decision-making. Pregnancies should not be terminated because of MDR/XDR-TB.

2) Aminoglycosides/polypeptides are not recommended for MDR/XDR-TB treatment during pregnancy.

3) Patients should be advised to maintain double barrier contraception during treatment of MDR/XDR-TB.

\section{Special considerations for MDR/XDR-TB and HIV co-infection}

The interaction between TB and HIV infection is characterised by a high probability of rapid progression to primary TB shortly after $M$. tuberculosis infection and from LTBI to overt disease in persons with preexisting HIV infection [238, 239].

Updated global drug-resistant TB surveillance data show that HIV-infected patients are more likely than HIV-uninfected cases to have MDR/XDR-TB, but this association is not statistically significant (pooled OR 1.4, 95\% CI 0.7-3.0; $\mathrm{p}=0.19$ ) and not consistent throughout the different regions surveyed [240]. In the future, eastern Europe may witness a large increase in the number of co-infected patients as it includes 15 out of 27 high MDR-TB burden countries [241] and, in contrast to other regions, both HIV incidence and AIDS-related deaths continue to increase [242]. In this region, it is of paramount importance that integrated TB, HIV, and drug substitution and needle exchange programmes are strengthened for civil society and the penitentiary system, in concert with harm- and stigma-reduction policies. Expansion of HIV testing is also a high priority in the region and worldwide.

There is firm evidence that HIV-infected persons with MDR/XDR-TB have higher mortality rates compared with HIV-seronegative cases. High mortality with extremely short survival was reported from South Africa $[243,244]$ among MDR/XDR-TB and HIV co-infected cases with very low CD4 cell counts and limited access to antiretroviral therapy (ART). HIV infection was an independent predictor of death (relative risk 4.22, 95\% CI 2.65-6.72) in patients treated with SLDs in Estonia, Latvia, the Philippines, Russia and Peru [245]. In Europe, MDR-TB cases had a poorer treatment outcome compared with non-MDR-TB among HIV-infected persons (27\% versus 44\%) [246]. However, recent reports from Latvia [247] and Russia [248] did not identify HIV as a predictor of death among MDR-TB cases, suggesting that other factors may be more important as determinants of TB treatment outcome in specific settings.

Timely diagnosis of MDR/XDR-TB among HIV-infected persons is crucial to reduce mortality: conventional DST methods have a turnaround time of a few weeks, which is unsuitable for immediate action. In HIV-infected patients, molecular tests allow a rate of case detection that is increased by $45 \%$, as compared with smear microscopy [249]. Xpert MTB/RIF is a rapid point-of-care molecular assay that is 
sensitive and specific for detection of TB when it is used as an initial diagnostic test in patients suspected of having HIV-associated TB [250]. WHO recommends Xpert MTB/RIF as a primary diagnostic test for TB in persons living with AIDS: the test should be made widely available in HIV-prevalent settings to improve patient care [251]. Rapid point-of-care diagnostic tests such as molecular assays should primarily be made available to HIV-infected communities and individuals [252].

The role of ART in reducing TB incidence, recurrence and mortality is well established. Limited data are available regarding ART initiation in MDR/XDR-TB patients. Evidence from 10 studies (including two European cohorts $[11,247]$ ) of HIV-infected patients using SLDs suggests a lower risk of death and a higher likelihood of TB cure in patients who were treated with ART versus those who were not [10]. Based on these data, WHO recommends that, among patients with MDR/XDR-TB, ART should be initiated as soon as possible (within the first 8 weeks) following anti-TB treatment initiation, irrespective of CD4 cell-count [241]. It may be speculated that, as demonstrated for TB/HIV co-infected persons with drug-susceptible M. tuberculosis, persons with $\mathrm{CD}^{+}{ }^{+} \mathrm{T}$-cell counts of $<50$ per cubic millimetre should start ART within 2 weeks of starting TB treatment in order to prevent new AIDS-defining illnesses and death [253, 254].

Systematic assessment of serious adverse events, treatment adherence, drug interactions and the incidence of immune reconstitution inflammation syndrome associated with SLDs in HIV-infected patients with MDR/ XDR-TB is rather scarce and needs to be improved. Drug absorption in HIV-infected patients can be significantly reduced. Drug concentration measurement can be performed to assure drug exposure (table 4).

While the evidence of the importance of acquired resistance among HIV-infected persons is elusive, the risk of primary disease after infection with drug-resistant strains is substantial, as demonstrated by the outbreaks of MDR-TB among hospitalised HIV-infected patients and healthcare workers in Europe and the USA [238]. Strategies for infection control based on administrative measures, environmental control and respiratory protection should become a priority in countries where both MDR/XDR-TB and HIV are prevalent. HIV-infected and -uninfected TB cases should be managed to similar standards, based on the principle that adequate adherence to standard regimens will prevent drug resistance in both situations [26].

\subsection{Conclusions}

1) There is a weak association between HIV infection and increased risk of MDR/XDR-TB, although numbers of patients with MDR/XDR-TB and HIV co-infection will likely rise in eastern European countries, as both conditions are increasingly prevalent in the area.

2) HIV-infected persons with MDR/XDR-TB have higher mortality rates compared with HIV-uninfected MDR/XDR-TB cases.

3) Timely diagnosis based on molecular assays is crucial to reduce the mortality associated with MDR/XDRTB patients among HIV-infected persons.

4) ART reduces the risk of death and increases the likelihood of TB cure in MDR/XDR-TB and HIV coinfected patients.

5) ART should be initiated as soon as possible (within the first 8 weeks) following anti-TB treatment initiation, irrespective of CD4 cell count, in patients with MDR/XDR-TB.

6) Strategies for infection control in hospitals and outpatient clinics should be regarded as a priority in countries where both MDR/XDR-TB and HIV are prevalent.

\section{Special considerations for patient management in areas of high MDR/XDR-TB prevalence}

MDR/XDR-TB increasingly occurs in economically disadvantaged regions of Europe [7]. Countries with a high burden of MDR/XDR-TB share common features of health systems that are not capable of coping with the challenges related to the emergence of drug-resistant TB, such as poverty and insufficient resources, inadequate technical equipment, shortage of medical education and training, lack of community support, and failing political commitment [255].

The high frequency of MDR-TB among new laboratory-confirmed cases in some countries of the WHO European region indicates active ongoing transmission of drug-resistant strains of M. tuberculosis [7]. Infection control measures to prevent the spread of MDR/XDR M. tuberculosis, especially in congregate settings like prisons and hospitals, are likely to be insufficient. The extent of possible nosocomial transmission is unclear, as molecular surveillance of $M$. tuberculosis strain transmission is not established in the high-burden countries. Exposure to patients who are infectious can be minimised by adequate infection control measures including reducing the time of hospitalisation, reducing the number of outpatient visits, 
avoiding overcrowding in wards and waiting areas, and prioritising community-care approaches for TB management, where possible [66, 256-261].

Delayed case detection and lack of access to rapid and accurate diagnosis of TB and M. tuberculosis DST are major obstacles for TB control in countries with high MDR/XDR-TB prevalence. Only 37\% of TB cases in the 18 high-priority countries in the European region are confirmed by culture and can be further evaluated by conventional DST [7]. This highlights the enormous gap in the identification of patients with MDR/ XDR-TB in the European Region. Knowledge of M. tuberculosis drug susceptibility/resistance pattern, particularly to SLDs, is especially important for the management of patients with MDR/XDR-TB in Europe, due to the increasing frequency of $M$. tuberculosis strains resistance to SLDs [36]. Scale-up for universal availability of diagnostic methods for the rapid TB identification and DST by molecular methods is an utmost priority to combat MDR/XDR-TB in Europe [262]. Moreover, universal access to standard (conventional/culture-based) first- and second-line DST is still essential as the current generation of molecular tests does not identify all relevant mutations that lead to phenotypic drug resistance [262].

Access to treatment, availability of quality-assured SLDs throughout therapy and continuous treatment monitoring are often lacking in countries with a high prevalence of MDR/XDR-TB in Europe. At present, only $\sim 20 \%$ of all estimated cases with MDR/XDR-TB in the European region receive adequate treatment with quality-assured drugs [16]. Although the Green Light Committee (GLC) and the Global Drug Facility are providing very important support for the sustained provision of essential anti-TB drugs in priority countries, political willingness of the countries to provide their own resources is essential and often insufficient. In addition, access to new drugs like linezolid, carbapenems, bedaquiline and clofazimine is not yet ensured by the GLC mechanism, although it is intended [263]. Availability of SLDs from quality-assured suppliers, regulated access and local governments must ensure distribution of drugs.

Default from treatment is a major problem in countries of high MDR/XDR-TB prevalence. Economic needs (e.g. migration for work) often overrule adherence and jeopardise treatment success.

Necessary interventions to address the enormous challenges for the prevention and management of MDR/ $\mathrm{XDR}-\mathrm{TB}$, especially in the high-burden countries of the WHO European Region are outlined in the Roadmap to Prevent and Combat Drug-Resistant Tuberculosis [16].

\subsection{Conclusions}

1) Rapid drug-resistant case detection, appropriate treatment, extended contact investigation, infection control measures and managing nonadherence leading to interruptions and defaults are the main components of MDR/XDR-TB case management.

2) It is essential that countries should have a strong monitoring and evaluation programme in place.

3) Investments in infrastructure, diagnostics and provision of care, effective infection control strategies, training, increased awareness, promotion of standards and allocation of appropriate resources are necessary.

\section{MDR/XDR-TB consilia}

Due to limited experience in the care of patients with MDR/XDR-TB even by infectious diseases or respiratory diseases specialists in many countries of the European region, there is a danger that these patients are managed in a less than optimum way. Sharing information and discussing clinical management among experts together with the caregivers in charge ensure better care for these patients.

In some European countries, there is a single consilium or committee to which all MDR/XDR-TB cases are reported and regularly discussed among experienced physicians, microbiologists and nurses. Data on drugsensitivity patterns are used to plan which drugs will be used in the regimen. As treatment progresses and new drug resistance patterns emerge or patients appear to be failing treatment, alternative regimens can be devised and implemented.

In this way MDR/XDR-TB patients are managed only by a small group of experts within the country, who can share their knowledge and build on it with further experience.

Regular meetings in a face-to-face consilium are not feasible for larger European countries. In this case, use of electronic information transfer for a centrally coordinated advisory service enables a small number of experts to give advice on patients about whom the service had been informed and to discuss the progress of each patient as required by clinical need. By central coordination, the aim of the service is to ensure that the best advice is given for every MDR/XDR-TB case in the country. A successful model for an electronic consilium has been established by the British Thoracic Society in cooperation with other relevant professional bodies [153], which is now being adapted in other countries. In 2012, WHO and ERS launched the ERS/WHO electronic consilium [160]. 


\subsection{Conclusions}

1) At present, there are no clear guidelines based on randomised controlled clinical trials to guide clinicians in the management of patients with MDR/XDR-TB.

2) Many clinicians may have relatively little experience in the management of MDR/XDR-TB and benefit from a professional exchange with colleagues to improve patient care.

3) Depending on the TB prevalence, local, regional or national consilia permit a pooling of expertise to provide the best patient management.

4) Consilia can be organised face-to-face or electronically. Patient data discussed in consilia must be handled according to data protection laws.

\section{Palliative care of patients with MDR/XDR-TB}

MDR/XDR-TB is a life-threatening condition in which most patients experience major physical and emotional suffering resulting from the disease and the treatment itself. This suffering does not always end with the end of successful treatment, as the disease quite often leaves long-term sequelae. Yet major suffering goes unnoticed in MDR/XDR-TB patients in whom all treatment options are exhausted. Although there are almost no systematic representative data describing the fate of these patients, clinical experience indicates in XDR-TB patients, there is high mortality within months, especially when they are co-infected with HIV, not on ART or within a few years after treatment failure is declared [264, 265].

As part of a comprehensive response to the epidemic of MDR/XDR-TB, there is an ethical obligation to alleviate suffering in patients through palliative care services. This has been increasingly recognised by TB and palliative care experts $[266,267]$.

Palliative care is defined as "an approach that improves the quality of life of patients and their families facing the problem associated with life-threatening illness, through the prevention and relief of suffering by means of early identification and impeccable assessment and treatment of pain and other problems, physical, psychosocial and spiritual” [268].

Data from the WHO Global TB Report 2011 suggest that the TB epidemic in several countries of eastern Europe is slowly being replaced by one of MDR-TB [266]. In 2011, the treatment success rate in the WHO European Region was only $48.5 \%$. This confirms the massive, largely unaddressed need for delivery of palliative care and end-of-life care to MDR/XDR-TB patients.

Existing expertise in palliative care should be sought and applied to MDR/XDR-TB cases. New expertise needs to be developed for carers for MDR/XDR-TB patients. TB infection control is a true challenge for patients and carers. General principles have to be applied. There is no consensus about continued drug treatment after declaration of treatment failure with no further options of cure.

Practical guidelines for palliative care in MDR/XDR- TB are available in South Africa [269].

In the Consolidated Action Plan to Prevent and Combat Multidrug- and Extensively Drug-Resistant Tuberculosis in the WHO European Region 2011-2015 [16], countries committed to establish, and strengthen as needed, palliative care mechanisms for MDR/XDR-TB patients who fail treatment, and to conduct operational research on service models (needs of patients, cost and resources) for provision of palliative care. Technical assistance on designing and running palliative care services in TB control programmes have to be urgently intensified.

\subsection{Conclusions}

1) $\mathrm{MDR} / \mathrm{XDR}-\mathrm{TB}$ care and control programmes should develop palliative care protocols, which should include pathways to access relevant medications including opioids to manage respiratory distress.

2) Access to palliative and end-of-life care services should be ensured to all patients with MDR/XDR-TB in need of it, including effective infection control measures at household or at the hospices where patients receive care.

\section{Consensus statements}

\subsection{How should contacts of patients with MDR/XDR-TB be managed?}

All close contacts of infectious MDR/XDR-TB patients should be screened for active TB and LTBI. Susceptible contacts, such as children $<5$ years of age and immunocompromised persons, and those with symptoms suggestive of TB, should be screened as soon as possible after diagnosis of the index patient. When active TB and TB infection have been excluded, screening should be repeated $\geqslant 8$ weeks after the last 
infectious exposure, to identify possible TST or IGRA conversion. At a minimum, infected contacts should be educated on signs and symptoms of TB and to seek medical evaluation when these occur.

Agreed: 39; disagreed: 1; abstained: 0 .

20.2 Should preventive chemotherapy be offered to all close contacts of patients with MDR/XDR-TB with a positive TST or IGRA test result in the absence of active TB?

Evidence for the effectiveness of preventive therapy of infected MDR/XDR-TB contacts is low. While, in principle, intention to test for LTBI is intention to treat with preventive chemotherapy, in contacts of patients with MDR/XDR-TB, intention to test is intention for close monitoring, as there is currently insufficient evidence to recommend preventive chemotherapy for MDR/XDR-TB contacts with LTBI in general. Preventive treatment should only be considered when a drug with bactericidal activity is available within the drug-sensitivity pattern of the M. tuberculosis culture from the source case and the risk of MDR/ XDR TB in the contact outweighs the risk of adverse events, particularly in young children and immunocompromised individuals.

Agreed: 39; disagreed: 0; abstained: 1 .

\subsection{What should be the choice of drugs and the length of preventive treatment?}

If a decision for preventive chemotherapy in MDR/XDR-TB contacts with LTBI is made, a fluoroquinolone (or other drug with bactericidal activity) should be used according to the M. tuberculosis DST result from the source case. A second drug should be added to protect the fluoroquinolone (or other drug with bactericidal activity) from the development of $M$. tuberculosis drug resistance, choosing among pyrazinamide, ethambutol or ethionamide/prothionamide according to the sensitivity pattern of the strain. The optimal duration of preventive chemotherapy in MDR/XDR-TB contacts with LTBI is not known. According to information available on the effectiveness and efficacy of preventive chemotherapy in patients with pan-susceptible strains of $M$. tuberculosis, the suggested duration of preventive chemotherapy with currently available medications should likely not be shorter than 9 months.

Agreed: 31; disagreed: 2; abstained: 7.

\subsection{What are the best infection control measures?}

The best way to prevent transmission of M. tuberculosis is a rapid diagnosis of TB and a prompt start of an effective treatment.

Infection control measures should be applied to all patients with a presumptive diagnosis of TB until contagiousness has been ruled out. Although isolation of patients with positive sputum smear microscopy results should be attempted, in daily practice, strict isolation of a patient is often not possible and infection control measures by hospital staff often cannot be strictly enforced.

To reduce the risk of $M$. tuberculosis transmission, natural ventilation should be maximised. Hospital staff sharing air with contagious patients with MDR/XDR-TB should wear FFP2-certified respirators. Contagious MDR/XDR-TB patients should be educated about the importance of cough hygiene and of infection control measures (e.g. wearing surgical masks outside of their room or when in contact with others in their room). Contagious MDR/XDR-TB patients should be confined to single-patient rooms, when possible. Upper room UVGI should be implemented in high-risk M. tuberculosis transmission areas as an effective infection control intervention.

Agreed: 39; disagreed: 0; abstained: 1 .

\subsection{How long are patients thought to be infectious and what needs to be considered prior to discharge from the hospital?}

Infectiousness is substantially reduced once a patient is on an adequate anti-TB treatment regimen. However, the optimal time for safe discharge from the hospital of patients with MDR/XDR-TB is currently not known. While the majority of experts in this consensus statement suggest hospitalisation of patients with MDR/XDR-TB until sputum cultures become negative, there is probably a trend to keep patients with MDR/XDR-TB too long in hospitals in many European countries. Importantly, exposure of convalescent and or immunocompromised patients to newly admitted infectious (smear-positive) patients needs to be avoided. Prior to the discharge of a patient with MDR/XDR-TB from the hospital, continuation of care and monitoring during the outpatient phase of treatment must be ensured. A practical checklist of issues that need to be addressed and solved before a hospitalised patient with MDR/XDR-TB should be discharged is provided in table 7 (see also online supplementary table S1).

Agreed: 39; disagreed: 0; abstained: 1 . 
20.6 Should molecular testing for MDR/XDR-TB (e.g. GeneXpert or MTBDRplus) be performed on sputum, bronchoalveolar lavage or extrapulmonary specimen in all persons with presumptive MDR/XDR-TB?

As molecular testing by automated nucleic acid amplification (e.g. GeneXpert) or LPAs (e.g. MTBDRplus) can rapidly identify patients with MDR-TB, these tests should be performed in all persons with presumptive MDR-TB, when available. Rapid diagnosis is crucial in HIV-infected individuals and in countries of high MDR-TB prevalence. In low MDR-TB prevalence countries, physicians must be aware of possible falsepositive resistance results of molecular tests and rifampicin resistance should be confirmed by in vitro antibiotic testing.

Agreed: 38 agree; disagreed: 1 ; abstained: 1 .

20.7 Should molecular drug resistance testing for XDR-TB (e.g. MTBDRsl) be performed on direct specimens or cultured specimens in all suspects of XDR-TB?

When available, in all patients with evidence of $M$. tuberculosis with an $r p o B$ mutation in a direct specimen or when DST indicates MDR-TB, molecular testing for second-line resistance (especially for fluoroquinolones and aminoglycosides/capreomycin) should be undertaken to reduce the time to diagnosis of XDR-TB. However, there is currently no consensus due to the absence of correlation between specific mutations and clinical outcome, whether the initial choice of a MDR/XDR-TB drug regimen, pending the results of culture DST, should be based on the results of rapid molecular drug-resistance diagnosis. If mutations in the M. tuberculosis genome are identified by molecular drug-resistance testing, physicians are strongly advised to discuss the likely clinical relevance of the identified mutations in a MDR/XDR-TB consilium, which includes expert microbiologists.

Agreed: 35; disagreed: 0; abstained: 5 .

20.8 When should anti-TB treatment be initiated in patients with positive results for $M$. tuberculosis suggestive of MDR-TB on rapid molecular testing (e.g. GeneXpert)?

In general, anti-TB treatment should be initiated immediately following the result of rapid molecular testing, when available. This is especially recommended for patients with TB meningitis, miliary TB and acute TB sepsis (Landouzy). In patients with sputum smear-negative TB, some experts suggest deferring the initiation of therapy until results from in vitro DST become available. There is currently no consensus of whether identification of rifampicin resistance in M. tuberculosis by GeneXpert or similar technology, suggesting MDR-TB, should be confirmed in a second independent specimen prior to the initiation of a standard MDR-TB treatment regimen in countries of low MDR/XDR-TB prevalence when the test is performed on patients with low risk of MDR-TB.

Agreed: 35; disagreed: 0; abstained: 5 .

\subsection{What should be the initial choice of drugs in MDR/XDR-TB treatment pending the results} of DST?

With a history of contact with MDR/XDR-TB, such patients should initially receive the same regimen as the putative index case. New patients without known contact with patients with MDR/XDR-TB or patients with previous treatment should initially receive the WHO standardised recommended SLD regimen consisting of five drugs, namely a fluoroquinolone (such as moxifloxacin or levofloxacin), a SLID, a thionamide (ethionamide or prothionamide), one more oral bacteriostatic drug (preferably cycloserine) plus pyrazinamide.

Agreed: 35; disagreed: 0; abstained: 5 .

20.10 What are the main principles of anti-TB drug treatment once DST results are available?

When the results from the culture DST are available, the treatment regimen should be individualised according to the DST result with at least four drugs that are likely to be effective. The drugs should be chosen in a hierarchical order according to their expected activity. Ethambutol and pyrazinamide should be included in any MDR/XDR-TB drug regimen, when the results of DST suggest that they might be useful. However, these drugs should not be counted among the four active drugs. For the other drugs, the order fluoroquinolone, SLID, prothionamide/ethionamide, cycloserine/terizidone, PAS, linezolid then other group 5 drugs is considered to be reasonable, although studies comparing the effectiveness of different regimens are missing. When available, linezolid should be considered as the first option of group 5 drugs for the treatment of MDR/XDR-TB. Most experts contributing to this document agree that clofazimine and meropenem/clavulanic acid may possibly be more effective for the treatment against XDR-TB than clarithromycin or amoxicillin/clavulanic acid, although again evidence is lacking.

Agreed: 36; disagreed: 1 ; abstained: 3 . 


\subsection{Should pyrazinamide be included in all MDR/XDR-TB treatment regimen?}

WHO has recommended use of pyrazinamide without pyrazinamide-susceptibility testing, which is technically difficult and often not performed. There is currently no consensus on whether patients with MDR/XDR-TB should be treated with pyrazinamide without pyrazinamide-susceptibility testing or when pyrazinamidesusceptibility testing has been performed but the test result indicates pyrazinamide drug resistance.

Agreed: 35; disagreed: 2; abstained: 3.

\subsection{Which monitoring investigations should be performed at what time during treatment?}

In addition to and prior to the initiation of MDR/XDR-TB therapy, baseline investigations should be performed including height and weight, audiometry and visual acuity testing, thoracic imaging, ECG, full blood count, liver, renal and thyroid function assessments, serological tests for co-infections such as HIV and viral hepatitis $\mathrm{B}$ and $\mathrm{C}$, and mycobacteriological investigations. Response to treatment and adverse drug events should be assessed and monitored at regular intervals (table 6). Sputum smear and culture examination should be performed at least once a month. In adults, time to culture conversion is a valuable measure of response to treatment.

Agreed: 35; disagreed: 0; abstained: 5 .

\subsection{How can adherence to therapy be supported and treatment success be ensured?}

Each patient should preferably have a special assigned healthcare worker, so that there is a single point of contact (e.g. for adverse effects, loss of tablets and change in social circumstances). In order to ensure that all patients receive the best possible treatment, the patient and the assigned healthcare worker should keep a record of each dose of drugs administered. Potential causes of nonadherence should be sought/identified and resolved. Recommendations for monitoring treatment response (smear, culture and imaging studies), screening for adverse events (audiogram and laboratory tests) and infection control precautions should follow a standardised schedule (table 6). Regular (e.g. 3-monthly) review of patients with MDR/XDR-TB by a multidisciplinary team (consilium) is helpful to ensure good communication among all the healthcare providers.

Agreed: 40; disagreed: 0; abstained: 0 .

\subsection{What is the optimal duration of anti-TB therapy in MDR/XDR-TB?}

The optimal duration of anti-TB therapy of MDR-TB is unknown, reflecting the limited evidence supporting current recommendations on this topic. Analysis of the largest data set in adults revealed that the optimal duration of the initial intensive phase was 7-8.4 months and the optimal total duration of therapy was 19-21 months in MDR-TB. Evidence-based recommendations for the duration of treatment for patients with XDR-TB are not available. Data in children are anecdotal. In the absence of evidence, recommendations for the duration of treatment in MDR-TB also apply currently to patients with XDR-TB. However, based on expert opinion, there may be situations where shorter regimens, particularly in the initial intensive phase, are appropriate. These include patients with less extensive disease, no prior treatment (especially with SLDs), and rapid conversion of sputum smears and cultures, as well as children.

Agreed: 36; disagreed: 2; abstained: 2 .

\subsection{What should be considered at the end of MDR/XDR-TB treatment?}

At the end of MDR/XDR-TB treatment outcome based on standardised definitions must be recorded and previous carers (hospital, consilium, etc.) and surveillance systems must be informed (table 8; for a complete checklist, see online supplementary table S1). End-of-treatment imaging is very helpful, as patients may be reassessed later for suspected relapse or reinfection. Patients must have clear information about signs and symptoms of recurrent TB. They should already receive a first follow-up appointment and should be actively followed for $\geqslant 2$ years.

Agreed: 40; disagreed: 0; abstained: 0 .

\section{Priorities for improvements of the management of patients with MDR/XDR-TB}

As is evident from the previous chapters, improvements in several areas, including basic research, capacity building and education, prevention, diagnosis, and treatment, are necessary to achieve the goal of TB eradication in times of emerging $M$. tuberculosis drug resistance.

\subsection{Provision of care}

Improving the care of patients as well as their clinical outcome must be the first priority for MDR/XDR-TB management. At present, $<20 \%$ of patients with MDR/XDR-TB have access to adequate therapy. Proper 
education and counselling about TB, especially MDR/XDR-TB, are important for patients, carers and healthcare providers. Contact with organisations that include patient representatives (e.g. www.takethattb.com) may offer support, hope and better insights for patients with MDR/XDR-TB. There are substantial gaps between the in-patient and outpatient care arrangements in many areas of Europe, including industrialised countries of the EU.

In many countries with a high burden of MDR/XDR-TB in the European Region, in-patients with TB are frequently cared for by physicians who are primarily specialised in the management of TB. While this ultimately results in great individual expertise, it also narrows the activities for such physicians to the point where it is perceived as unattractive for their long-term career development. As a consequence, only a limited number of possibly older physicians will be available for MDR/XDR-TB care in these countries in the future. Merging departments of TB and respiratory medicine and, therefore, widening the scope of expertise for young physicians in training can address this problem.

\subsection{Prevention}

In the absence of a better vaccine, advances in MDR/XDR-TB prevention will have to rely on improvements in infection control strategies and better identification of persons latently infected with MDR/XDR strains of M. tuberculosis. Effective treatment regimens of shorter duration to prevent MDR/XDR-TB in those latently infected are not available at present, and the choice of existing drugs and the duration of preventive chemotherapy for MDR/XDR-TB are unclear. The evidence base for length of treatment of active MDR/ $\mathrm{XDR}-\mathrm{TB}$ in children and how to deal with exposed and possibly infected paediatric contacts remains poor at present. Clinical trials are now urgently required to evaluate different regimens for preventive chemotherapy of MDR/XDR-TB in adults and children. Such studies will have to include many centres in order to achieve sufficient statistical power, if absence of disease/relapse at a given time-point serves as the primary endpoint. Ideally, these studies should also be conducted in a variety of epidemiological settings across Europe, as outcome might be influenced by re-exposure and re-infection in high-burden environments. TBNET offers a good infrastructure to conduct such studies.

\subsection{Diagnostics}

Although molecular diagnostics have substantially improved the rapid diagnosis of MDR/XDR-TB in recent years, these currently lack the necessary sensitivity in individuals with negative sputum smears (e.g. in children, smear-negative and/or HIV-infected adults, and in patients with extrapulmonary TB). At present, it is unclear to what extent the identification of specific mutations in the genome of M. tuberculosis relates to second-line drug resistance and should influence the decision of the choice of drugs for the treatment of patients with TB. Molecular DST is still far from being a reality. In vitro drug resistance testing by phenotypic or molecular methods for some of the second-line TB drugs and novel TB drugs needs to be established, standardised and correlated to the in vivo efficacy of these medications in MDR/XDR-TB treatment.

\subsection{Treatment}

One of the substantial differences between the management of patients with MDR/XDR-TB and patients with other bacterial infections is the very long duration of antibiotic therapy necessary to achieve relapsefree cure. Biomarkers are urgently needed as surrogate markers for relapse-free cure to individualise MDR/ XDR-TB therapies, and to monitor progression and response to therapy.

Treatment for MDR/XDR-TB is far from optimal at present. Shorter treatment regimes with higher efficacy and better tolerability than those currently available are required. As the development of new drugs for the treatment of TB has been neglected for decades, only a few compounds have currently reached the clinical stage of evaluation. New drugs for the treatment of MDR/XDR-TB need to be developed. In addition, reevaluation of existing drugs for anti-TB properties should be performed to harness their potential efficacy in the treatment of MDR/XDR-TB. Novel generations of diagnostics and new anti-TB drugs need evaluation at clinical trial sites in high-prevalence areas, but clinical research on MDR/XDR-TB in high-prevalence countries in Europe is underrepresented. Thoughtful cooperation among basic and clinical researchers, clinical trial groups and professional societies, industry, nongovernmental organisations, and other stakeholders should build infrastructure, knowledge and resources for good-quality research in Europe.

\subsection{Conclusions}

There is no doubt that intensified research efforts in the field of MDR/XDR-TB are needed. Without substantial improvements in the provision of care, in the development of diagnostics, in finding better treatment regimens and especially in the area of TB prevention, the goal of "zero TB deaths, zero new TB infections, zero TB suffering" cannot be achieved. At a time when financial resources may be shrinking globally, it is particularly important to establish research coalitions and cross-disciplinary teams of experts 
in order to improve the evidence base, which ought to guide our clinical practice and maximise the successful management of MDR/XDR-TB even in the poorest settings.

\section{Acknowledgements}

The authors' affiliations are as follows. C. Lange: Division of Clinical Infectious Diseases, Medical Clinic, Research Center Borstel, German Center for Infection Research, Borstel, Germany, and Dept of Medicine, University of Namibia School of Medicine, Windhoek, Namibia. I. Abubakar; Research Dept of Infections and Population Health, and MRC Clinical Trials Unit, University College London, London, UK. J-W.C. Alffenaar: University of Groningen, University Medical Center Groningen, Dept of Clinical Pharmacy and Pharmacology, Groningen, The Netherlands. G. Bothamley: Homerton University Hospital, London, UK. J.A. Caminero: Dept of Pneumology, University Hospital of Gran Canaria "Dr.Negrín", Las Palmas de Gran Canaria, Spain, and Multidrug-Resistant Tuberculosis Unit, Tuberculosis and HIV Dept, International Union against Tuberculosis and Lung Disease (the Union), Paris, France. A.C.C. Carvalho: Clinic of Infectious and Tropical Diseases, WHO Collaborating Centre for TB/HIV Co-infection, University of Brescia, Brescia, Italy, and Laboratory of Innovations in Therapies, Education and Bioproducts (LITEB), Oswaldo Cruz Institute (IOC), Rio de Janeiro, Brazil. K-C. Chang: Tuberculosis and Chest Service, Dept of Health, Hong Kong, China. L. Codecasa: Regional TB Reference Centre, Villa Marelli Institute, Niguarda Ca'Granda Hospital, Milan, Italy. A. Correia: Public Health Dept, Portuguese Northern Health Administration, Porto, Portugal. V. Crudu: Phthisiopneumology Institute, Chisinau, Republic of Moldova. P. Davies: Liverpool Heart and Chest Hospital, Liverpool, UK. M. Dedicoat: Heart of England Foundation Trust, Birmingham, and University of Warwick, Coventry, UK. F. Drobniewski: Public Health England National Mycobacterial Reference Laboratory and Imperial College, London, UK. R. Duarte: Centro Hospitalar de Vila Nova de Gaia/Espinho, Portuguese Reference Centre for M/XDR-TB, and Dept of Clinical Epidemiology, Predictive Medicine and Public Health, Institute of Public Health University of Porto, Porto, Portugal. C. Ehlers: Take That TB, Hamburg, Germany. C. Erkens: KNCV Tuberculosis Foundation, The Hague, The Netherlands. D. Goletti: Translational Research Unit, Dept of Epidemiology and Preclinical Research, "L. Spallanzani" National Institute for Infectious Diseases (INMI), Rome, Italy. G. Günther: Division of Clinical Infectious Diseases, Medical Clinic, Research Center Borstel, German Center for Infection Research, Borstel, Germany, and Dept of Medicine, University of Namibia School of Medicine, Windhoek, Namibia. E. Ibraim: Marius Nasta Institute of Pulmonology, Bucharest, Romania. B. Kampmann: Dept of Paediatrics, Imperial College, London, UK, and Vaccinology Theme, MRC Unit, The Gambia. L. Kuksa: Riga East University Hospital, Tuberculosis and Lunge Disease Center, Riga, Latvia. W. de Lange: Tuberculosis Center Beatrixoord, University Medical Center Groningen, Groningen, The Netherlands. F. van Leth: Dept of Global Health, Academic Medical Center, University of Amsterdam, and Amsterdam Institute for Global Health and Development, Amsterdam, The Netherlands. J. van Lunzen: Infectious Diseases Unit, University Medical Center Hamburg, Hamburg, Germany. A. Matteelli: Clinic of Infectious and Tropical Diseases, WHO Collaborating Centre for TB/HIV Co-infection, University of Brescia, Brescia, Italy. D. Menzies: Montreal Chest Institute, McGill University, Montreal, Canada. I. Monedero: Multidrug-Resistant Tuberculosis Unit, Tuberculosis and HIV Dept, The Union, Paris, France, and Hospital Serveis Clinics, Barcelona, Spain. E. Richter: National Reference Center for Mycobacteria, Research Center Borstel, Borstel, Germany. S. Rüsch-Gerdes: National Reference Center for Mycobacteria, Research Center Borstel, Borstel, Germany. A. Sandgren: European Centre for Disease Prevention and Control (ECDC), Stockholm, Sweden. A. Scardigli: Multidrug-Resistant Tuberculosis Unit, Tuberculosis and HIV Dept, The Union, Paris, France. A. Skrahina: Republican Research and Practical Centre for Pulmonology and Tuberculosis, Minsk, Belarus. E. Tortoli: Emerging Bacterial Pathogens Unit, San Raffaele Scientific Institute, Milan, Italy. G. Volchenkov: Vladimir Regional TB Dispensary, Center of Excellence for TB Infection Control, Vladimir, Russia. D. Wagner: Center for Infectious Diseases, and Center for Chronic Immunodeficiency, University Medical Center Freiburg, Freiburg, Germany. M.J. van der Werf: ECDC, Stockholm, Sweden. B. Williams: Dept of Paediatrics, North West London Hospitals NHS Trust, UK. W-W. Yew: Stanley Ho Centre for Emerging Infectious Diseases, The Chinese University of Hong Kong, Hong Kong, China. J-P. Zellweger: Swiss Lung Association, Berne, Switzerland. D.M. Cirillo: Emerging Bacterial Pathogens Unit, San Raffaele Scientific Institute, Milan, Italy.

The authors' contributions were as follows. Chapter 1: C. Lange; chapter 2: C. Lange (chapter leader) and D.M. Cirillo; chapter 3: M.J. van der Werf; chapter 4: C. Lange; chapter 5: J-P. Zellweger (chapter leader) and F. van Leth; chapter 6: C. Erkens (chapter leader), I. Abubakar, B. Kampmann and A. Sandgren; chapter 7: W. de Lange (chapter leader) and G. Volchenkov; chapter 8: D.M. Cirillo (chapter leader), F. Drobniewski, E. Richter, S. Rüsch-Gerdes and E. Tortoli; chapter 9: J.A. Caminero (chapter leader), J-W. Alffenaar, K-C. Chang, I. Monedero, A. Scardigli and W.W. Yew; chapter 10: D. Menzies; chapter 11: M. Dedicoat (chapter leader) and P. Davies; chapter 12: G. Bothamley (chapter leader), G. Günther and D. Wagner; chapter 13: R. Duarte (chapter leader), L. Codecasa and A. Correia; chapter 14: B. Kampmann (chapter leader) and B. Williams; chapter 15: G. Günther (chapter leader) and C. Lange; chapter 16: A. Matteelli (chapter leader), A.C.C. Carvalho, D. Goletti and J. van Lunzen; chapter 17: E. Ibraim (chapter leader), V. Crudu and A. Skrahina; chapter 18: P. Davies; chapter 19: L. Kuksa (chapter leader), G. Günther and C. Lange; chapter 20: C. Lange (chapter leader), G. Bothamley, J.A. Caminero, D.M. Cirillo, P. Davies, R. Duarte, C. Erkens, E. Ibraim, W. de Lange, A. Matteelli, D. Menzies, M.J. van der Werf and J-P. Zellweger; chapter 21: D.M. Cirillo (chapter leader), C. Lange, C. Ehlers, B. Kampmann and W.W. Yew.

The authors express their gratitude to Dennis Falzon (WHO, Geneva, Switzerland) who contributed to the chapter 3, Masoud Dara, Kristin Kremer and Andrei Dadu (all WHO Europe, Copenhagen, Denmark) who contributed to the chapter 4, and Ernesto Jaramillo (WHO, Geneva, Switzerland) who contributed to chapter 19. We are also thankful for the technical advice of Martina Sester (Saarland University, Homburg, Germany), and technical assistance by Susanne Grossmann (Research Center Borstel, Borstel, Germany) and Sandra Ramouta (Montreal Chest Institute, Montreal, Canada).

\section{References}

1 Lim SS, Vos T, Flaxman AD, et al. A comparative risk assessment of burden of disease and injury attributable to 67 risk factors and risk factor clusters in 21 regions, 1990-2010: a systematic analysis for the Global Burden of Disease Study 2010. Lancet 2012; 380: 2224-2260. 
World Health Organization. Global tuberculosis report 2013. Geneva, Switzerland, 2013.

Skrahina A, Hurevich H, Zalutskaya A, et al. Alarming levels of drug-resistant tuberculosis in Belarus: results of a survey in Minsk. Eur Respir J 2012; 39: 1425-1431.

4 Skrahina A, Hurevich H, Zalutskaya A, et al. Multidrug-resistant tuberculosis in Belarus: the size of the problem and associated risk factors. Bull World Health Organ 2013; 91: 36-45.

5 Falzon D, Gandhi N, Migliori GB, et al. Resistance to fluoroquinolones and second-line injectable drugs: impact on multidrug-resistant TB outcomes. Eur Respir J 2013; 42: 156-168.

6 Orenstein EW, Basu S, Shah NS, et al. Treatment outcomes among patients with multidrug-resistant tuberculosis: systematic review and meta-analysis. Lancet Infect Dis 2009; 9: 153-161.

7 European Centre for Disease Prevention and Control, WHO Regional Office for Europe. Tuberculosis surveillance and monitoring in Europe. Stockholm, European Centre for Disease Prevention and Control, 2013.

8 Lassen O. On the curability of open pulmonary tuberculosis. Acta Tuberc Scand 1950; 26: 169-170.

9 East African/British Medical Research Council. Results at 5 years of a controlled comparison of a 6-month and a standard 18-month regimen of chemotherapy for pulmonary tuberculosis. Am Rev Respir Dis 1977; 116: 3-8.

10 World Health Organization. Guidelines for the programmatic management of drug-resistant tuberculosis. www. who.int/tb/challenges/mdr/programmatic_guidelines_for_mdrtb/en/ Date last accessed: December 12, 2012. Date last updated: 2011.

11 Soldatou A, Davies EG. Respiratory virus infections in the immunocompromised host. Paediatr Respir Rev 2003; 4: 193-204.

12 Diel R, Rutz S, Castell S, et al. Tuberculosis: cost of illness in Germany. Eur Respir J 2012; 40: 143-151.

13 Boehme CC, Nabeta P, Hillemann D, et al. Rapid molecular detection of tuberculosis and rifampin resistance. N Engl J Med 2010; 363: 1005-1015.

14 Greco S, Girardi E, Navarra A, et al. Current evidence on diagnostic accuracy of commercially based nucleic acid amplification tests for the diagnosis of pulmonary tuberculosis. Thorax 2006; 61: 783-790.

15 Food and Drug Administration. FDA news release. www.fda.gov/NewsEvents/Newsroom/PressAnnouncements/ ucm333695.htm Date last accessed: 2012. Date last updated: December 31, 2012.

16 World Health Organization. Roadmap to prevent and combat drug-resistant tuberculosis. www.euro.who.int/ data/assets/pdf_file/0014/152015/e95786.pdf Date last updated: 2011.

17 European Centre for Disease Control and Prevention. Management of contacts of MDR TB and XDR TB patients. Stockholm, Sweden, 2012.

18 World Health Organization. Definitions and reporting framework for tuberculosis - 2013 revision. Geneva, Switzerland, 2013.

19 Velayati AA, Masjedi MR, Farnia P, et al. Emergence of new forms of totally drug-resistant tuberculosis bacilli: super extensively drug-resistant tuberculosis or totally drug-resistant strains in Iran. Chest 2009; 136: 420-425.

20 Udwadia ZF, Amale RA, Ajbani KK, et al. Totally drug-resistant tuberculosis in India. Clin Infect Dis 2012; 54: 579-581.

21 Pai M, O’Brien R. New diagnostics for latent and active tuberculosis: state of the art and future prospects. Semin Respir Crit Care Med 2008; 29: 560-568.

22 World Health Organization. Stop TB Department of WHO technical consultation in Geneva on 21-22 March 2012. www.who.int/tb/challenges/xdr/xdrconsultation/en/index.html Date last accessed: 2012. Date last updated: March, 2012.

23 World Health Organization. Guidelines for the programmatic management of drug-resistant tuberculosis. WHO, Geneva, 2008.

24 World Health Organization. Management of MDR-TB: a field guide: a companion document to guidelines for programmatic management of drug-resistant tuberculosis: integrated management of adolescent and adult illness (IMAI). http://apps.who.int/iris/handle/10665/44163?locale=es Date last accessed: December 13, 2012. Date last updated: 2009.

25 International Union Against Tuberculosis and Lung Diseases. Guidelines for Clinical and Operational Management of Drug-Resistant Tuberculosis. IUTLD, Paris, 2013.

26 Migliori GB, Zellweger JP, Abubakar I, et al. European Union Standards for Tuberculosis Care. Eur Respir J 2012; 39: 807-819.

27 Tuberculosis Coalition for Technical Assistance. International Standards for Tuberculosis Care. ISTC, The Hague, 2009.

28 Erkens CG, Kamphorst M, Abubakar I, et al. Tuberculosis contact investigation in low prevalence countries: a European consensus. Eur Respir J 2010; 36: 925-949.

29 Centers of Diseases Control and Prevention. Guidelines for the investigation of contacts of persons with infectious tuberculosis. Recommendations from the National Tuberculosis Controllers Association and CDC. MMWR Recomm Rep 2005; 54: 1-47.

30 Targeted tuberculin testing and treatment of latent tuberculosis infection. Am J Respir Crit Care Med 2000; 161: S221-S247.

31 World Health Organization. Towards universal access to diagnosis and treatment of multidrug-resistant and extensively drug-resistant tuberculosis by 2015. www.who.int/tb/publications/2011/mdr_report_2011/en/ Date last accessed: December 12, 2012. Date last updated: March 23, 2011.

32 World Health Organization. Global tuberculosis report 2012. Geneva, Switzerland, 2012.

33 Faustini A, Hall AJ, Perucci CA. Risk factors for multidrug resistant tuberculosis in Europe: a systematic review. Thorax 2006; 61: 158-163.

34 Liang L, Wu Q, Gao L, et al. Factors contributing to the high prevalence of multidrug-resistant tuberculosis: a study from China. Thorax 2012; 67: 632-638.

35 Shen X, DeRiemer K, Yuan ZA, et al. Drug-resistant tuberculosis in Shanghai, China, 2000-2006: prevalence, trends and risk factors. Int J Tuberc Lung Dis 2009; 13: 253-259.

36 Dalton T, Cegielski P, Akksilp S, et al. Prevalence of and risk factors for resistance to second-line drugs in people with multidrug-resistant tuberculosis in eight countries: a prospective cohort study. Lancet 2012; 380: 1406-1417.

37 Shin SS, Keshavjee S, Gelmanova IY, et al. Development of extensively drug-resistant tuberculosis during multidrug-resistant tuberculosis treatment. Am J Respir Crit Care Med 2010; 182: 426-432.

38 Brewer TF, Choi HW, Seas C, et al. Self-reported risks for multiple-drug resistance among new tuberculosis cases: implications for drug susceptibility screening and treatment. PLoS One 2011; 6: e25861. 
39 Otero L, Krapp F, Tomatis C, et al. High prevalence of primary multidrug resistant tuberculosis in persons with no known risk factors. PLoS One 2011; 6: e26276.

40 Balabanova Y, Radiulyte B, Davidaviciene E, et al. Risk factors for drug-resistant tuberculosis patients in Lithuania, 2002-2008. Eur Respir J 2012; 39: 1266-1269.

41 Zhao Y, Xu S, Wang L, et al. National survey of drug-resistant tuberculosis in China. N Engl J Med 2012; 366: 2161-2170.

42 van der Werf MJ, Langendam MW, Huitric E, et al. Multidrug resistance after inappropriate tuberculosis treatment: a meta-analysis. Eur Respir J 2012; 39: 1511-1519.

43 World Health Organization. Multidrug and extensively drug-resistant TB (M/XDR-TB) 2010 Global Report on Surveillance and response. Geneva, Switzerland, 2010.

44 Lan NTN, Lien HTK, Tung LB, et al. Mycobacterium tuberculosis Beijing genotype and risk for treatment failure and relapse, Vietnam. Emerg Infect Dis 2003; 9: 1633-1635.

45 Bate R, Jensen P, Hess K, et al. Substandard and falsified anti-tuberculosis drugs: a preliminary field analysis. Int J Tuberc Lung Dis 2013; 17: 308-311.

46 Pasipanodya JG, Mcllleron H, Burger A, et al. Serum drug concentrations predictive of pulmonary tuberculosis outcomes. J Infect Dis 2013; 208: 1464-1473.

47 Royce S, Falzon D, van Weezenbeek C, et al. Multidrug resistance in new tuberculosis patients: burden and implications. Int J Tuberc Lung Dis 2013; 17: 511-513.

48 Stuckler D, Basu S, McKee M, et al. Mass incarceration can explain population increases in TB and multidrugresistant TB in European and central Asian countries. Proc Natl Acad Sci USA 2008; 105: 13280-13285.

49 Shin SS, Keshavjee S, Gelmanova IY, et al. Development of extensively drug-resistant tuberculosis during multidrug-resistant tuberculosis treatment. Am J Respir Crit Care Med 2010; 182: 426-432.

50 Minion J, Gallant V, Wolfe J, et al. Multidrug and extensively drug-resistant tuberculosis in Canada 1997-2008: demographic and disease characteristics. PLoS One 2013; 8: e53466.

$51 \mathrm{Hu} \mathrm{Y,} \mathrm{Mathema} \mathrm{B,} \mathrm{Jiang} \mathrm{W,} \mathrm{et} \mathrm{al.} \mathrm{Transmission} \mathrm{pattern} \mathrm{of} \mathrm{drug-resistant} \mathrm{tuberculosis} \mathrm{and} \mathrm{its} \mathrm{implication} \mathrm{for}$ tuberculosis control in eastern rural China. PLoS One 2011; 6: e19548.

52 Fox GJ, Dobler CC, Marks GB. Active case finding in contacts of people with tuberculosis. Cochrane Database Syst Rev 2011; 9: CD008477.

53 Behr MA, Hopewell PC, Paz EA, et al. Predictive value of contact investigation for identifying recent transmission of Mycobacterium tuberculosis. Am J Respir Crit Care Med 1998; 158: 465-469.

54 Cox H, van Cutsem G. Household screening and multidrug-resistant tuberculosis. Lancet 2011; 377: 103-104.

55 Becerra MC, Appleton SC, Franke MF, et al. Tuberculosis burden in households of patients with multidrugresistant and extensively drug-resistant tuberculosis: a retrospective cohort study. Lancet 2011; 377: 147-152.

Veen J. Microepidemics of tuberculosis: the stone-in-the-pond principle. Tuber Lung Dis 1992; 73: 73-76.

57 Vynnycky E, Fine PE. Lifetime risks, incubation period, and serial interval of tuberculosis. Am J Epidemiol 2000; 152: 247-263.

58 Poulsen A. Some clinical features of tuberculosis. 1. Incubation period. Acta Tuberc Scand 1950; 24: 311-346.

59 Fraser A, Paul M, Attamna A, et al. Drugs for preventing tuberculosis in people at risk of multiple-drug-resistant pulmonary tuberculosis. Cochrane Database Syst Rev 2009; 2: CD005435.

60 van der Werf MJ, Langendam MW, Sandgren A, et al. Lack of evidence to support policy development for management of contacts of multidrug-resistant tuberculosis patients: two systematic reviews. Int J Tuberc Lung Dis 2012; 16: 288-296.

61 Schaaf HS, Gie RP, Kennedy M, et al. Evaluation of young children in contact with adult multidrug-resistant pulmonary tuberculosis: a 30-month follow-up. Pediatrics 2002; 109: 765-771.

62 Denholm JT, Leslie DE, Jenkin GA, et al. Long-term follow-up of contacts exposed to multidrug-resistant tuberculosis in Victoria, Australia, 1995-2010. Int J Tuberc Lung Dis 2012; 16: 1320-1325.

63 Two simultaneous outbreaks of multidrug-resistant tuberculosis - Federated States of Micronesia, 2007-2009. MMWR Morb Mortal Wkly Rep 2009; 58: 253-256.

64 van der Werf MJ, Sandgren A, Manissero D. Management of contacts of multidrug-resistant tuberculosis patients in the European Union and European Economic Area. Int J Tuberc Lung Dis 2012; 16: 426.

65 Schaaf HS, Marais BJ. Management of multidrug-resistant tuberculosis in children: a survival guide for paediatricians. Paediatr Respir Rev 2011; 12: 31-38.

66 Falzon D, Jaramillo E, Wares F, et al. Universal access to care for multidrug-resistant tuberculosis: an analysis of surveillance data. Lancet Infect Dis 2013; 13: 690-697.

67 World Health Organization. WHO policy on TB infection control in health-care facilities, congregate settings and households. www.who.int/tb/publications/2009/9789241598323/en/ Date last accessed: December 13, 2012. Date last updated: 2009.

68 Jensen PA, Lambert LA, Iademarco MF, et al. Guidelines for preventing the transmission of Mycobacterium tuberculosis in health-care settings, 2005. MMWR Recomm Rep 2005; 54: 1-141.

69 TB CARE I. Infection Control, www.tbcare1.org/publications/toolbox/ic

70 Drobniewski F, Balabanova Y, Nikolayevsky V, et al. Drug-resistant tuberculosis, clinical virulence, and the dominance of the Beijing strain family in Russia. JAMA 2005; 293: 2726-2731.

71 Borrell S, Gagneux S. Infectiousness, reproductive fitness and evolution of drug-resistant Mycobacterium tuberculosis. Int J Tuberc Lung Dis 2009; 13: 1456-1466.

72 Riley RL, Mills CC, Nyka W, et al. Aerial dissemination of pulmonary tuberculosis. A two-year study of contagion in a tuberculosis ward. 1959. Am J Epidemiol 1995; 142: 3-14.

73 Riley RL, Mills CC, O'Grady F, et al. Infectiousness of air from a tuberculosis ward. Ultraviolet irradiation of infected air: comparative infectiousness of different patients. Am Rev Respir Dis 1962; 85: 511-525.

74 Rouillon A, Perdrizet S, Parrot R. Transmission of tubercle bacilli: the effects of chemotherapy. Tubercle 1976; 57: 275-299.

75 Behr MA, Warren SA, Salamon H, et al. Transmission of Mycobacterium tuberculosis from patients smear-negative for acid-fast bacilli. Lancet 1999; 353: 444-449.

76 Escombe AR, Moore DA, Gilman RH, et al. The infectiousness of tuberculosis patients coinfected with HIV. PLoS Med 2008; 5: e188. 
Fennelly KP, Jones-Lopez EC, Ayakaka I, et al. Variability of infectious aerosols produced during coughing by patients with pulmonary tuberculosis. Am J Respir Crit Care Med 2012; 186: 450-457.

78 Fitzwater SP, Caviedes L, Gilman RH, et al. Prolonged infectiousness of tuberculosis patients in a directly observed therapy short-course program with standardized therapy. Clin Infect Dis 2010; 51: 371-378.

Sepkowitz KA. How contagious is tuberculosis? Clin Infect Dis 1996; 23: 954-962.

80 Menzies D. Effect of treatment on contagiousness of patients with active pulmonary tuberculosis. Infect Control Hosp Epidemiol 1997; 18: 582-586.

81 Coffey CC, Lawrence RB, Campbell DL, et al. Fitting characteristics of eighteen N95 filtering-facepiece respirators. J Occup Environ Hyg 2004; 1: 262-271.

82 US Department of Labor. Occupational and Health Safety Standards 1910 AppA: Fit Testing Procedures. Washington, Department of Labor, 2004

83 Dharmadhikari AS, Mphahlele M, Stoltz A, et al. Surgical face masks worn by patients with multidrug-resistant tuberculosis: impact on infectivity of air on a hospital ward. Am J Respir Crit Care Med 2012; 185: 1104-1109.

84 Bock NN, Jenssen PA, Nardell E. Tuberculosis infection control in resource-limited settings in the era of expanding HIV care and treatment. J Infect Dis 2007; 196: Suppl. 1, S108-S113.

85 World Health Organization. Natural ventilation for infection control in health-care settings. WHO guideline 2009. Geneva, Switzerland, 2009.

86 Streifel AJ. Design and maintenance of hospital ventilation systems and prevention of airborne nosocomial infections. In: Mayhall CG, ed. Hospital Epidemiology and Infection Control. 2nd Edn. Philadelphia, Lippincott Williams and Wilkins, 1999; pp. 1211-1221.

87 Centers for Disease Control and Prevention. Environmental Control for Tuberculosis: Basic Upper-Room Ultraviolet Germicidal Irradiation. Atlanta, Guidelines for Healthcare Settings, 2009.

$88 \mathrm{Xu}$ P, Kujundzic E, Peccia J, et al. Impact of environmental factors on efficacy of upper-room air ultraviolet germicidal irradiation for inactivating airborne mycobacteria. Environ Sci Technol 2005; 39: 9656-9664.

89 Lee K, Slavcev A, Nicas M. Respiratory protection against Mycobacterium tuberculosis: quantitative fit test outcomes for five type N95 filtering-facepiece respirators. J Occup Environ Hyg 2004; 1: 22-28.

90 Balabanova Y, Drobniewski F, Nikolayevskyy V, et al. An integrated approach to rapid diagnosis of tuberculosis and multidrug resistance using liquid culture and molecular methods in Russia. PLoS One 2009; 4: e7129.

91 World Health Organization. The use of liquid TB culture and drug susceptibility testing (DST) in low and medium income settings. Geneva, Switzerland, 2007.

92 Pfyffer GE, Bonato DA, Ebrahimzadeh A, et al. Multicenter laboratory validation of susceptibility testing of Mycobacterium tuberculosis against classical second-line and newer antimicrobial drugs by using the radiometric BACTEC 460 technique and the proportion method with solid media. J Clin Microbiol 1999; 37: 3179-3186.

93 Rusch-Gerdes S, Pfyffer GE, Casal M, et al. Multicenter laboratory validation of the BACTEC MGIT 960 technique for testing susceptibilities of Mycobacterium tuberculosis to classical second-line drugs and newer antimicrobials. J Clin Microbiol 2006; 44: 688-692.

94 Kruuner A, Yates MD, Drobniewski FA. Evaluation of MGIT 960-based antimicrobial testing and determination of critical concentrations of first- and second-line antimicrobial drugs with drug-resistant clinical strains of Mycobacterium tuberculosis. J Clin Microbiol 2006; 44: 811-818.

95 Kim SJ, Espinal MA, Abe C, et al. Is second-line anti-tuberculosis drug susceptibility testing reliable? Int $J$ Tuberc Lung Dis 2004; 8: 1157-1158.

96 Sulochana S, Venkataraman P, Paramasivan CN. Evaluation of various methods of susceptibility to ofloxacin in strains of Mycobacterium tuberculosis. Indian J Med Res 1999; 110: 186-189.

97 Somasundaram S, Paramasivan NC. Susceptibility of Mycobacterium tuberculosis strains to gatifloxacin and moxifloxacin by different methods. Chemotherapy 2006; 52: 190-195.

98 Alvirez-Freites EJ, Carter JL, Cynamon MH. In vitro and in vivo activities of gatifloxacin against Mycobacterium tuberculosis. Antimicrob Agents Chemother 2002; 46: 1022-1025.

99 Rodrigues C, Jani J, Shenai S, et al. Drug susceptibility testing of Mycobacterium tuberculosis against second-line drugs using the Bactec MGIT 960 System. Int J Tuberc Lung Dis 2008; 12: 1449-1455.

100 Almeida Da Silva PE, Palomino JC. Molecular basis and mechanisms of drug resistance in Mycobacterium tuberculosis: classical and new drugs. J Antimicrob Chemother 2011; 66: 1417-1430.

101 Traore H, Fissette K, Bastian I, et al. Detection of rifampicin resistance in Mycobacterium tuberculosis isolates from diverse countries by a commercial line probe assay as an initial indicator of multidrug resistance. Int J Tuberc Lung Dis 2000; 4: 481-484.

102 Ramaswamy S, Musser JM. Molecular genetic basis of antimicrobial agent resistance in Mycobacterium tuberculosis: 1998 update. Tuber Lung Dis 1998; 79: 3-29.

103 Zhang Y, Heym B, Allen B, et al. The catalase-peroxidase gene and isoniazid resistance of Mycobacterium tuberculosis. Nature 1992; 358: 591-593.

104 Laurenzo D, Mousa SA. Mechanisms of drug resistance in Mycobacterium tuberculosis and current status of rapid molecular diagnostic testing. Acta Trop 2011; 119: 5-10.

105 Larsen MH, Vilcheze C, Kremer L, et al. Overexpression of inhA, but not kasA, confers resistance to isoniazid and ethionamide in Mycobacterium smegmatis, M. bovis BCG and M. tuberculosis. Mol Microbiol 2002; 46: 453-466.

106 van Deun A, Maug AK, Salim MA, et al. Short, highly effective, and inexpensive standardized treatment of multidrug-resistant tuberculosis. Am J Respir Crit Care Med 2010; 182: 684-692.

107 Bottger EC. The ins and outs of Mycobacterium tuberculosis drug susceptibility testing. Clin Microbiol Infect 2011; 17: 1128-1134.

108 Plinke C, Rusch-Gerdes S, Niemann S. Significance of mutations in embB codon 306 for prediction of ethambutol resistance in clinical Mycobacterium tuberculosis isolates. Antimicrob Agents Chemother 2006; 50: $1900-1902$.

109 Miotto P, Cabibbe AM, Mantegani P, et al. GenoType MTBDRsl performance on clinical samples with diverse genetic background. Eur Respir J 2012; 40: 690-698.

110 Zhang Y, Yew WW. Mechanisms of drug resistance in Mycobacterium tuberculosis. Int J Tuberc Lung Dis 2009; 13: 1320-1330.

111 Aubry A, Pan XS, Fisher LM, et al. Mycobacterium tuberculosis DNA gyrase: interaction with quinolones and correlation with antimycobacterial drug activity. Antimicrob Agents Chemother 2004; 48: 1281-1288. 
112 Von Groll A, Martin A, Jureen P, et al. Fluoroquinolone resistance in Mycobacterium tuberculosis and mutations in gyrA and gyrB. Antimicrob Agents Chemother 2009; 53: 4498-4500.

113 Via LE, Cho SN, Hwang S, et al. Polymorphisms associated with resistance and cross-resistance to aminoglycosides and capreomycin in Mycobacterium tuberculosis isolates from South Korean Patients with drug-resistant tuberculosis. J Clin Microbiol 2010; 48: 402-411.

114 Georghiou SB, Magana M, Garfein RS, et al. Evaluation of genetic mutations associated with Mycobacterium tuberculosis resistance to amikacin, kanamycin and capreomycin: a systematic review. PLoS One 2012; 7: e33275.

115 Van Deun A, Aung KJ, Bola V, et al. Rifampin drug resistance tests for tuberculosis: challenging the gold standard. J Clin Microbiol 2013; 51: 2633-2640.

116 Crudu V, Stratan E, Romancenco E, et al. First evaluation of an improved assay for molecular genetic detection of tuberculosis as well as rifampin and isoniazid resistances. J Clin Microbiol 2012; 50: 1264-1269.

117 Boehme CC, Nicol MP, Nabeta P, et al. Feasibility, diagnostic accuracy, and effectiveness of decentralised use of the Xpert MTB/RIF test for diagnosis of tuberculosis and multidrug resistance: a multicentre implementation study. Lancet 2011; 377: 1495-1505.

118 World Health Organization. Policy statement: automated real-time nucleic acid amplification technology for rapid and simultaneous detection of tuberculosis and rifampicin resistance: Xpert MTB/RIF system. Geneva, World Health Organization, 2011.

119 Tortoli E, Russo C, Piersimoni C, et al. Clinical validation of Xpert MTB/RIF for the diagnosis of extrapulmonary tuberculosis. Eur Respir J 2012; 40: 442-447.

120 Caminero JA, Sotgiu G, Zumla A, et al. Best drug treatment for multidrug-resistant and extensively drug-resistant tuberculosis. Lancet Infect Dis 2010; 10: 621-629.

121 Johnson JL, Hadad DJ, Boom WH, et al. Early and extended early bactericidal activity of levofloxacin, gatifloxacin and moxifloxacin in pulmonary tuberculosis. Int J Tuberc Lung Dis 2006; 10: 605-612.

122 Rustomjee R, Lienhardt C, Kanyok T, et al. A Phase II study of the sterilising activities of ofloxacin, gatifloxacin and moxifloxacin in pulmonary tuberculosis. Int J Tuberc Lung Dis 2008; 12: 128-138.

123 Caminero JA. A tuberculosis guide for specialist physicians. Paris, International Union Against Tuberculosis and Lung Disease, 2004.

124 Caminero JA. Treatment of multidrug-resistant tuberculosis: evidence and controversies. Int J Tuberc Lung Dis 2006; 10: 829-837.

125 Caminero JA, Matteelli A, Lange C. Treatment of TB. In: Lange C, Migliori GB, eds. Tuberculosis. Eur Respir Monogr 2012; 58: 154-166.

126 Falzon D, Jaramillo E, Schunemann HJ, et al. WHO guidelines for the programmatic management of drug-resistant tuberculosis: 2011 update. Eur Respir J 2011; 38: 516-528.

127 Caminero JA. Management of multidrug-resistant tuberculosis and patients in retreatment. Eur Respir J 2005; 25: 928-936.

128 Kim SJ. Drug-susceptibility testing in tuberculosis: methods and reliability of results. Eur Respir J 2005; 25: 564-569.

129 van Deun A, Martin A, Palomino JC. Diagnosis of drug-resistant tuberculosis: reliability and rapidity of detection. Int J Tuberc Lung Dis 2010; 14: 131-140.

130 Jonsson S, Davidse A, Wilkins J, et al. Population pharmacokinetics of ethambutol in South African tuberculosis patients. Antimicrob Agents Chemother 2011; 55: 4230-4237.

131 Donald PR, Maritz JS, Diacon AH. Pyrazinamide pharmacokinetics and efficacy in adults and children. Tuberculosis (Edinb) 2012; 92: 1-8.

132 Donald PR, Sirgel FA, Venter A, et al. The early bactericidal activity of amikacin in pulmonary tuberculosis. Int $J$ Tuberc Lung Dis 2001; 5: 533-538.

133 Peloquin CA, Berning SE, Nitta AT, et al. Aminoglycoside toxicity: daily versus thrice-weekly dosing for treatment of mycobacterial diseases. Clin Infect Dis 2004; 38: 1538-1544.

134 Donomae I. Capreomycin in the treatment of pulmonary tuberculosis. Ann NY Acad Sci 1966; 135: 1011-1038.

135 Peloquin CA, Hadad DJ, Molino LP, et al. Population pharmacokinetics of levofloxacin, gatifloxacin, and moxifloxacin in adults with pulmonary tuberculosis. Antimicrob Agents Chemother 2008; 52: 852-857.

136 Pranger AD, van Altena R, Aarnoutse RE, et al. Evaluation of moxifloxacin for the treatment of tuberculosis: 3 years of experience. Eur Respir J 2011; 38: 888-894.

137 Lee HW, Kim DW, Park JH, et al. Pharmacokinetics of prothionamide in patients with multidrug-resistant tuberculosis. Int J Tuberc Lung Dis 2009; 13: 1161-1166.

138 Zhu M, Namdar R, Stambaugh JJ, et al. Population pharmacokinetics of ethionamide in patients with tuberculosis. Tuberculosis (Edinb) 2002; 82: 91-96.

139 Donald PR, Sirgel FA, Venter A, et al. Early bactericidal activity of amoxicillin in combination with clavulanic acid in patients with sputum smear-positive pulmonary tuberculosis. Scand J Infect Dis 2001; 33: 466-469.

140 Gopal M, Padayatchi N, Metcalfe JZ, et al. Systematic review of clofazimine for the treatment of drug-resistant tuberculosis. Int J Tuberc Lung Dis 2013; 17: 1001-1007.

141 Alffenaar JW, van Altena R, Harmelink IM, et al. Comparison of the pharmacokinetics of two dosage regimens of linezolid in multidrug-resistant and extensively drug-resistant tuberculosis patients. Clin Pharmacokinet 2010; 49: 559-565.

142 McGee B, Dietze R, Hadad DJ, et al. Population pharmacokinetics of linezolid in adults with pulmonary tuberculosis. Antimicrob Agents Chemother 2009; 53: 3981-3984.

143 Pasipanodya J, Gumbo T. An oracle: antituberculosis pharmacokinetics-pharmacodynamics, clinical correlation, and clinical trial simulations to predict the future. Antimicrob Agents Chemother 2011; 55: 24-34.

144 Gumbo T, Dona CS, Meek C, et al. Pharmacokinetics-pharmacodynamics of pyrazinamide in a novel in vitro model of tuberculosis for sterilizing effect: a paradigm for faster assessment of new antituberculosis drugs. Antimicrob Agents Chemother 2009; 53: 3197-3204.

145 Rodriguez JC, Cebrian L, Lopez M, et al. Mutant prevention concentration: comparison of fluoroquinolones and linezolid with Mycobacterium tuberculosis. J Antimicrob Chemother 2004; 53: 441-444.

146 Shandil RK, Jayaram R, Kaur P, et al. Moxifloxacin, ofloxacin, sparfloxacin, and ciprofloxacin against Mycobacterium tuberculosis: evaluation of in vitro and pharmacodynamic indices that best predict in vivo efficacy. Antimicrob Agents Chemother 2007; 51: 576-582. 
147 Gumbo T, Louie A, Deziel MR, et al. Selection of a moxifloxacin dose that suppresses drug resistance in Mycobacterium tuberculosis, by use of an in vitro pharmacodynamic infection model and mathematical modeling. J Infect Dis 2004; 190: 1642-1651.

148 Wallis RS, Jakubiec W, Kumar V, et al. Biomarker-assisted dose selection for safety and efficacy in early development of PNU-100480 for tuberculosis. Antimicrob Agents Chemother 2011; 55: 567-574.

149 Cynamon $\mathrm{MH}$, Zhang Y, Harpster $\mathrm{T}$, et al. High-dose isoniazid therapy for isoniazid-resistant murine Mycobacterium tuberculosis infection. Antimicrob Agents Chemother 1999; 43: 2922-2924.

150 Katiyar SK, Bihari S, Prakash S, et al. A randomised controlled trial of high-dose isoniazid adjuvant therapy for multidrug-resistant tuberculosis. Int J Tuberc Lung Dis 2008; 12: 139-145.

151 Koh WJ, Kwon OJ, Gwak H, et al. Daily $300 \mathrm{mg}$ dose of linezolid for the treatment of intractable multidrugresistant and extensively drug-resistant tuberculosis. J Antimicrob Chemother 2009; 64: 388-391.

152 Chang KC, Yew WW, Tam CM, et al. WHO group 5 drugs and difficult multidrug-resistant tuberculosis: a systematic review with cohort analysis and meta-analysis. Antimicrob Agents Chemother 2013; 57: 4097-4104.

153 Jordan TS, Cullen D, Davies PD. A centralised electronic Multidrug-Resistant Tuberculosis Advisory Service: the first 2 years. Int J Tuberc Lung Dis 2012; 16: 950-954.

154 Sotgiu G, Centis R, D'Ambrosio L, et al. Efficacy, safety and tolerability of linezolid containing regimens in treating MDR-TB and XDR-TB: systematic review and meta-analysis. Eur Respir J 2012; 40: 1430-1442.

155 Singla R, Caminero JA, Jaiswal A, et al. Linezolid: an effective, safe and cheap drug for patients failing multidrugresistant tuberculosis treatment in India. Eur Respir J 2012; 39: 956-962.

156 Chang KC, Yew WW, Cheung SW, et al. Can intermittent dosing optimize prolonged linezolid treatment of difficult multidrug-resistant tuberculosis? Antimicrob Agents Chemother 2013; 57: 3445-3449.

157 Dey $\mathrm{T}$, Brigden $\mathrm{G}$, Cox $\mathrm{H}$, et al. Outcomes of clofazimine for the treatment of drug-resistant tuberculosis: a systematic review and meta-analysis. J Antimicrob Chemother 2013; 68: 284-293.

158 Cholo MC, Steel HC, Fourie PB, et al. Clofazimine: current status and future prospects. J Antimicrob Chemother 2012; 67: 290-298.

159 De Lorenzo S, Alffenaar JW, Sotgiu G, et al. Efficacy and safety of meropenem-clavulanate added to linezolidcontaining regimens in the treatment of MDR-/XDR-TB. Eur Respir J 2013; 41: 1386-1392.

160 Blasi F, Dara M, van der Werf MJ, et al. Supporting TB clinicians managing difficult cases: the ERS/WHO Consilium. Eur Respir J 2013; 41: 491-494.

161 Yew WW, Lange C, Leung CC. Treatment of tuberculosis: update 2010. Eur Respir J 2011; 37: 441-462.

162 Ma Z, Lienhardt C, McIlleron H, et al. Global tuberculosis drug development pipeline: the need and the reality. Lancet 2010; 375: 2100-2109.

163 Caminero JA, Matteelli A, Loddenkemper R. Tuberculosis: are we making it incurable? Eur Respir J 2013; 42: 5-8.

164 Lienhardt C, Raviglione M, Spigelman M, et al. New drugs for the treatment of tuberculosis: needs, challenges, promise, and prospects for the future. J Infect Dis 2012; 205: Suppl. 2, S241-249.

165 Zumla A, Nahid P, Cole ST. Advances in the development of new tuberculosis drugs and treatment regimens. Nat Rev Drug Discov 2013; 12: 388-404.

166 Gler MT, Skripconoka V, Sanchez-Garavito E, et al. Delamanid for multidrug-resistant pulmonary tuberculosis. N Engl J Med 2012; 366: 2151-2160.

167 Diacon AH, Dawson R, Hanekom M, et al. Early bactericidal activity of delamanid (OPC-67683) in smear-positive pulmonary tuberculosis patients. Int J Tuberc Lung Dis 2011; 15: 949-954.

168 Diacon AH, Dawson R, von Groote-Bidlingmaier F, et al. 14-day bactericidal activity of PA-824, bedaquiline, pyrazinamide, and moxifloxacin combinations: a randomised trial. Lancet 2012; 380: 986-993.

169 Skripconoka V, Danilovits M, Pehme L, et al. Delamanid improves outcomes and reduces mortality in multidrugresistant tuberculosis. Eur Respir J 2013; 41: 1393-1400.

170 European Medicines Agency. European Medicines Agency recommends two new treatment options for tuberculosis. www.ema.europa.eu/ema/index.jsp?curl=pages/news_and_events/news/2013/11/news_detail_001972. jsp\&mid=WC0b01ac058004d5c1 Date last accessed: December 29, 2013. Date last updated: November 22, 2013.

171 Diacon AH, Pym A, Grobusch M, et al. The diarylquinoline TMC207 for multidrug-resistant tuberculosis. N Engl J Med 2009; 360: 2397-2405.

172 Iseman MD, Madsen L, Goble M, et al. Surgical intervention in the treatment of pulmonary disease caused by drugresistant Mycobacterium tuberculosis. Am Rev Respir Dis 1990; 141: 623-625.

173 Kempker RR, Vashakidze S, Solomonia N, et al. Surgical treatment of drug-resistant tuberculosis. Lancet Infect Dis 2012; 12: 157-166.

174 Toman K. Tuberculosis case-finding and chemotherapy. Questions and answers. Geneva, World Health Organization, 1979.

175 Rieder HL. Interventions for tuberculosis control and elimination. Paris, International Union Against Tuberculosis and Lung Disease, 2002; pp. 1-251.

176 Fox W. The current status of short-course chemotherapy. Tubercle 1979; 60: 177-190.

177 Fox W, Ellard GA, Mitchison DA. Studies on the treatment of tuberculosis undertaken by the British Medical Research Council tuberculosis units, 1946-1986, with relevant subsequent publications. Int J Tuberc Lung Dis 1999; 3: Suppl. 2, S231-S279.

178 Ahuja SD, Ashkin D, Avendano M, et al. Multidrug resistant pulmonary tuberculosis treatment regimens and patient outcomes: an individual patient data meta-analysis of 9,153 patients. PLoS Med 2012; 9: e1001300.

179 Loddenkemper R, Sotgiu G, Mitnick CD. Cost of tuberculosis in the era of multidrug resistance: will it become unaffordable? Eur Respir J 2012; 40: 9-11.

180 Dorman SE, Johnson JL, Goldberg S, et al. Substitution of moxifloxacin for isoniazid during intensive phase treatment of pulmonary tuberculosis. Am J Respir Crit Care Med 2009; 180: 273-280.

181 Leung EC, Yew WW, Leung CC, et al. Shorter treatment duration for selected patients with multidrug-resistant tuberculosis. Eur Respir J 2011; 38: 227-230.

182 Nunn A. The evaluation of a standardised treatment regimen of anti-tuberculosis drugs for patients with multidrug-resistant tuberculosis (MDR-TB), www.controlled-trials.com/ISRCTN78372190 Date last updated: November 19, 2102. 
183 Brown J, Dooley D, Backer H. Drug-resistant tuberculosis: a survival guide for clinicians. 2nd Edn. San Francisco, Curry International Tuberculosis Center, 2011.

184 Bloss E, Kuksa L, Holtz TH, et al. Adverse events related to multidrug-resistant tuberculosis treatment, Latvia, 2000-2004. Int J Tuberc Lung Dis 2010; 14: 275-281.

185 Yew WW, Leung CC. Antituberculosis drugs and hepatotoxicity. Respirology 2006; 11: 699-707.

186 Lehloenya RJ, Dheda K. Cutaneous adverse drug reactions to anti-tuberculosis drugs: state of the art and into the future. Expert Rev Anti Infect Ther 2012; 10: 475-486.

187 Kass JS, Shandera WX. Nervous system effects of antituberculosis therapy. CNS Drugs 2010; 24: 655-667.

188 de Jager P, van Altena R. Hearing loss and nephrotoxicity in long-term aminoglycoside treatment in patients with tuberculosis. Int J Tuberc Lung Dis 2002; 6: 622-627.

189 Talbert Estlin KA, Sadun AA. Risk factors for ethambutol optic toxicity. Int Ophthalmol 2010; 30: 63-72.

190 Xu HB, Jiang RH, Li L, et al. Linezolid in the treatment of MDR-TB: a retrospective clinical study. Int J Tuberc Lung Dis 2012; 16: 358-363.

191 McDonnell ME, Braverman LE, Bernardo J. Hypothyroidism due to ethionamide. N Engl J Med 2005; 352: 2757-2759.

192 Fortun J, Martin-Davila P, Navas E, et al. Cambios en la Epidemiología de la Tuberculosis: la Influencia de los movimientos migratorios internacionales [Changes in the epidemiology of tuberculosis: the influence of international migration flows]. Enferm Infecc Microbiol Clin 2011; 29: 654-659.

193 Pedrazzoli D, Fulton N, Anderson L, et al. Tuberculosis in the UK: 2012 report. London, Health Protection Agency, 2012.

194 Bonacci RA, Cruz-Hervert LP, Garcia-Garcia L, et al. Impact of cigarette smoking on rates and clinical prognosis of pulmonary tuberculosis in Southern Mexico. J Infect 2013; 66: 303-312.

195 Martineau AR, Timms PM, Bothamley GH, et al. High-dose vitamin $\mathrm{D}_{3}$ during intensive-phase antimicrobial treatment of pulmonary tuberculosis: a double-blind randomised controlled trial. Lancet 2011; 377: 242-250.

196 Perez-Guzman C, Vargas MH, Quinonez F, et al. A cholesterol-rich diet accelerates bacteriologic sterilization in pulmonary tuberculosis. Chest 2005; 127: 643-651.

197 van der Watt JJ, Harrison TB, Benatar M, et al. Polyneuropathy, anti-tuberculosis treatment and the role of pyridoxine in the HIV/AIDS era: a systematic review. Int J Tuberc Lung Dis 2011; 15: 722-728.

198 Eker B, Ortmann J, Migliori GB, et al. Multidrug- and extensively drug-resistant tuberculosis, Germany. Emerg Infect Dis 2008; 14: 1700-1706.

199 Diacon AH, Maritz JS, Venter A, et al. Time to detection of the growth of Mycobacterium tuberculosis in MGIT 960 for determining the early bactericidal activity of antituberculosis agents. Eur J Clin Microbiol Infect Dis 2010; 29: $1561-1565$.

200 Wejse C, Gustafson P, Nielsen J, et al. TBscore: Signs and symptoms from tuberculosis patients in a low-resource setting have predictive value and may be used to assess clinical course. Scand J Infect Dis 2008; 40: 111-120.

201 Malla P, Kanitz EE, Akhtar M, et al. Ambulatory-based standardized therapy for multi-drug resistant tuberculosis: experience from Nepal, 2005-2006. PLoS One 2009; 4: e8313.

202 Heller T, Lessells RJ, Wallrauch CG, et al. Community-based treatment for multidrug-resistant tuberculosis in rural KwaZulu-Natal, South Africa. Int J Tuberc Lung Dis 2010; 14: 420-426.

203 Mitnick C, Bayona J, Palacios E, et al. Community-based therapy for multidrug-resistant tuberculosis in Lima, Peru. N Engl J Med 2003; 348: 119-128.

204 Brust JC, Shah NS, Scott M, et al. Integrated, home-based treatment for MDR-TB and HIV in rural South Africa: an alternate model of care. Int J Tuberc Lung Dis 2012; 16: 998-1004.

205 Brannan JW. Home treatment of tuberculosis by means of hospital dispensaries. Trans Am Climatol Assoc 1904; 20: 149-163.

206 Toczek A, Cox H, du Cros P, et al. Strategies for reducing treatment default in drug-resistant tuberculosis: systematic review and meta-analysis. Int J Tuberc Lung Dis 2013; 17: 299-307.

207 Goemaere E, Ford N, Berman D, et al. XDR-TB in South Africa: detention is not the priority. PLoS Med 2007; 4: el62.

208 Bekker LG, Wood R. Community-based management of multidrug-resistant tuberculosis in South Africa. Int J Tuberc Lung Dis 2010; 14: 379.

209 Lutge EE, Wiysonge CS, Knight SE, et al. Material incentives and enablers in the management of tuberculosis. Cochrane Database Syst Rev 2012; 1: CD007952.

210 Cayla JA, Orcau A. Control of tuberculosis in large cities in developed countries: an organizational problem. $B M C$ Med 2011; 9: 127.

211 Hales CM, Heilig CM, Chaisson R, et al. The association between symptoms and microbiologically defined response to tuberculosis treatment. Ann Am Thorac Soc 2013; 10: 18-25.

212 Zar HJ, Connell TG, Nicol M. Diagnosis of pulmonary tuberculosis in children: new advances. Expert Rev Anti Infect Ther 2010; 8: 277-288.

213 Starke JR. Pediatric tuberculosis: time for a new approach. Tuberculosis (Edinb) 2003; 83: 208-212.

214 Dominguez J, De Souza-Galvao M, Ruiz-Manzano J, et al. T-cell responses to the Mycobacterium tuberculosisspecific antigens in active tuberculosis patients at the beginning, during, and after antituberculosis treatment. Diagn Microbiol Infect Dis 2009; 63: 43-51.

215 Marais BJ, Hesseling AC, Gie RP, et al. The bacteriologic yield in children with intrathoracic tuberculosis. Clin Infect Dis 2006; 42: e69-e71.

216 Gandhi NR, Nunn P, Dheda K, et al. Multidrug-resistant and extensively drug-resistant tuberculosis: a threat to global control of tuberculosis. Lancet 2010; 375: 1830-1843.

217 Seddon JA, Hesseling AC, Godfrey-Faussett P, et al. Risk factors for infection and disease in child contacts of multidrug-resistant tuberculosis: a cross-sectional study. BMC Infect Dis 2013; 13: 392.

218 Feja K, McNelley E, Tran CS, et al. Management of pediatric multidrug-resistant tuberculosis and latent tuberculosis infections in New York City from 1995 to 2003. Pediatr Infect Dis J 2008; 27: 907-912.

219 Flood J, Oh P, Lewis B, et al. Multidrug resistance among persons with tuberculosis in California, 1994-2003. JAMA 2005; 293: 2732-2739.

220 Mendez Echevarria A, Baquero Artigao F, Garcia Miguel MJ, et al. Tuberculosis mutirresistente en la edad pediatrica [Multidrug-resistant tuberculosis in the pediatric age group]. An Pediatr (Barc) 2007; 67: 206-211.

221 Drobac PC, Mukherjee JS, Joseph JK, et al. Community-based therapy for children with multidrug-resistant tuberculosis. Pediatrics 2006; 117: 2022-2029. 
222 Seddon JA, Hesseling AC, Willemse M, et al. Culture-confirmed multidrug-resistant tuberculosis in children: clinical features, treatment, and outcome. Clin Infect Dis 2012; 54: 157-166.

223 Fairlie L, Beylis NC, Reubenson G, et al. High prevalence of childhood multi-drug resistant tuberculosis in Johannesburg, South Africa: a cross sectional study. BMC Infect Dis 2011; 11: 28.

224 Schaaf HS, Gie RP, Kennedy M, et al. Evaluation of young children in contact with adult multidrug-resistant pulmonary tuberculosis: a 30-month follow-up. Pediatrics 2002; 109: 765-771.

225 Teixeira L, Perkins MD, Johnson JL, et al. Infection and disease among household contacts of patients with multidrug-resistant tuberculosis. Int J Tuberc Lung Dis 2001; 5: 321-328.

226 Kritski AL, Marques MJ, Rabahi MF, et al. Transmission of tuberculosis to close contacts of patients with multidrug-resistant tuberculosis. Am J Respir Crit Care Med 1996; 153: 331-335.

227 Ettehad D, Schaaf HS, Seddon JA, et al. Treatment outcomes for children with multidrug-resistant tuberculosis: a systematic review and meta-analysis. Lancet Infect Dis 2012; 12: 449-456.

228 Al-Dabbagh M, Lapphra K, McGloin R, et al. Drug-resistant tuberculosis: pediatric guidelines. Pediatr Infect Dis J 2011; 30: 501-505.

229 Seddon JA, Furin JJ, Gale M, et al. Caring for children with drug-resistant tuberculosis: practice-based recommendations. Am J Respir Crit Care Med 2012; 186: 953-964.

230 Palacios E, Dallman R, Munoz M, et al. Drug-resistant tuberculosis and pregnancy: treatment outcomes of 38 cases in Lima, Peru. Clin Infect Dis 2009; 48: 1413-1419.

231 Shin S, Guerra D, Rich M, et al. Treatment of multidrug-resistant tuberculosis during pregnancy: a report of 7 cases. Clin Infect Dis 2003; 36: 996-1003.

232 Lessnau KD, Qarah S. Multidrug-resistant tuberculosis in pregnancy: case report and review of the literature. Chest 2003; 123: 953-956.

233 Tabarsi P, Moradi A, Baghaei P, et al. Standardised second-line treatment of multidrug-resistant tuberculosis during pregnancy. Int J Tuberc Lung Dis 2011; 15: 547-550.

234 Donald PR, Doherty E, Van Zyl FJ. Hearing loss in the child following streptomycin administration during pregnancy. Cent Afr J Med 1991; 37: 268-271.

235 Loebstein R, Addis A, Ho E, et al. Pregnancy outcome following gestational exposure to fluoroquinolones: a multicenter prospective controlled study. Antimicrob Agents Chemother 1998; 42: 1336-1339.

236 Good JT Jr, Iseman MD, Davidson PT, et al. Tuberculosis in association with pregnancy. Am J Obstet Gynecol 1981; 140: 492-498.

237 Getahun H, Sculier D, Sismanidis C, et al. Prevention, diagnosis, and treatment of tuberculosis in children and mothers: evidence for action for maternal, neonatal, and child health services. J Infect Dis 2012; 205: Suppl. 2, S216-S227.

238 Wells CD, Cegielski JP, Nelson LJ, et al. HIV infection and multidrug-resistant tuberculosis: the perfect storm. J Infect Dis 2007; 196: Suppl. 1, S86-S107.

239 Matteelli A, Richardson MD, Sotgiu G, et al. Multidrug- and extensively drug-resistant TB in persons living with HIV. Expert Rev Respir Med 2009; 3: 245-254.

240 Zignol M, van Gemert W, Falzon D, et al. Surveillance of anti-tuberculosis drug resistance in the world: an updated analysis, 2007-2010. Bull World Health Organ 2012; 90: 111-119D.

241 World Health Organization. Global Tuberculosis Control 2011. Geneva, Switzerland, 2011.

242 UNAIDS. Global HIV/AIDS response. Epidemic update and health sector progress towards Universal Access. Progress report 2011. http://whqlibdoc.who.int/publications/2011/9789241502986_eng.pdf

243 Gandhi NR, Moll A, Sturm AW, et al. Extensively drug-resistant tuberculosis as a cause of death in patients coinfected with tuberculosis and HIV in a rural area of South Africa. Lancet 2006; 368: 1575-1580.

244 Gandhi NR, Shah NS, Andrews JR, et al. HIV coinfection in multidrug- and extensively drug-resistant tuberculosis results in high early mortality. Am J Respir Crit Care Med 2010; 181: 80-86.

245 Kurbatova EV, Taylor A, Gammino VM, et al. Predictors of poor outcomes among patients treated for multidrugresistant tuberculosis at DOTS-plus projects. Tuberculosis (Edinb) 2012; 92: 397-403.

246 Griffith DE, Aksamit T, Brown-Elliott BA, et al. An official ATS/IDSA statement: diagnosis, treatment, and prevention of nontuberculous mycobacterial diseases. Am J Respir Crit Care Med 2007; 175: 367-416.

247 Leimane V, Dravniece G, Riekstina V, et al. Treatment outcome of multidrug/extensively drug-resistant tuberculosis in Latvia, 2000-2004. Eur Respir J 2010; 36: 584-593.

248 Balabanova Y, Nikolayevskyy V, Ignatyeva O, et al. Survival of civilian and prisoner drug-sensitive, multi- and extensive drug- resistant tuberculosis cohorts prospectively followed in Russia. PLoS One 2011; 6: e20531.

249 Lawn SD, Kerkhoff AD, Vogt M, et al. Characteristics and early outcomes of patients with Xpert MTB/RIF-negative pulmonary tuberculosis diagnosed during screening before antiretroviral therapy. Clin Infect Dis 2012; 54: 1071-1079.

250 Steingart KR, Sohn H, Schiller I, et al. Xpert ${ }_{\circledast}$ MTB/RIF assay for pulmonary tuberculosis and rifampicin resistance in adults. Cochrane Database Syst Rev 2013; 1: CD009593.

251 World Health Organization. Xpert MTB/RIF increases timely TB detection among people living with HIV and saves lives. Information note. www.who.int/tb/challenges/hiv/Xpert_TBHIV_Information_Note_final.pdf Date last updated: 2013

252 Rachow A, Zumla A, Heinrich N, et al. Rapid and accurate detection of Mycobacterium tuberculosis in sputum samples by Cepheid Xpert MTB/RIF assay - a clinical validation study. PLoS One 2011; 6: e20458.

253 Havlir DV, Kendall MA, Ive P, et al. Timing of antiretroviral therapy for HIV-1 infection and tuberculosis. N Engl J Med 2011; 365: 1482-1491.

254 Abdool Karim SS, Naidoo K, Grobler A, et al. Integration of antiretroviral therapy with tuberculosis treatment. N Engl J Med 2011; 365: 1492-1501.

255 Abubakar I, Zignol M, Falzon D, et al. Drug-resistant tuberculosis: time for visionary political leadership. Lancet Infect Dis 2013; 13: 529-539.

256 Gelmanova IY, Keshavjee S, Golubchikova VT, et al. Barriers to successful tuberculosis treatment in Tomsk, Russian Federation: non-adherence, default and the acquisition of multidrug resistance. Bull World Health Organ 2007; 85: 703-711.

257 Gelmanova IY, Taran DV, Mishustin SP, et al. "Sputnik": a programmatic approach to improve tuberculosis treatment adherence and outcome among defaulters. Int J Tuberc Lung Dis 2011; 15: 1373-1379. 
Isaakidis $\mathrm{P}$, Cox HS, Varghese B, et al. Ambulatory multi-drug resistant tuberculosis treatment outcomes in a cohort of HIV-infected patients in a slum setting in Mumbai, India. PLoS One 2011; 6: e28066.

Fitzpatrick C, Floyd K. A systematic review of the cost and cost effectiveness of treatment for multidrug-resistant tuberculosis. Pharmacoeconomics 2012; 30: 63-80.

Suarez PG, Floyd K, Portocarrero J, et al. Feasibility and cost-effectiveness of standardised second-line drug treatment for chronic tuberculosis patients: a national cohort study in Peru. Lancet 2002; 359: 1980-1989.

Tupasi TE, Gupta R, Quelapio MI, et al. Feasibility and cost-effectiveness of treating multidrug-resistant tuberculosis: a cohort study in the Philippines. PLoS Med 2006; 3: e352.

2 Wells WA, Boehme CC, Cobelens FG, et al. Alignment of new tuberculosis drug regimens and drug susceptibility testing: a framework for action. Lancet Infect Dis 2013; 13: 449-458.

World Health Organization. Meeting report of the 3rd Global GLC meeting 17-19 October 2012. Geneva, Switzerland, 2012.

Dheda K, Shean K, Zumla A, et al. Early treatment outcomes and HIV status of patients with extensively drugresistant tuberculosis in South Africa: a retrospective cohort study. Lancet 2010; 375: 1798-1807.

5 O’Donnell MR, Padayatchi N, Kvasnovsky C, et al. Treatment outcomes for extensively drug-resistant tuberculosis and HIV co-infection. Emerg Infect Dis 2013; 19: 416-424.

Connor S, Foley K, Harding R, et al. Declaration on palliative care and MDR/XDR-TB. Int J Tuberc Lung Dis 2012; 16: 712-713.

Harding R, Foley KM, Connor SR, et al. Palliative and end-of-life care in the global response to multidrug-resistant tuberculosis. Lancet Infect Dis 2012; 12: 643-646.

World Health Organization. WHO Definition of Palliative Care. www.who.int/cancer/palliative/definition/en/ Hospice Palliative Care Association. Guidelines for Providing Palliative Care to Patients with Tuberculosis. www. hospicepalliativecaresa.co.za/pdf/patientcare/TB_Guidelines_2011.PDF Date last updated: May 2011. 